10.

\title{
Über Curven dritter Ordnung und die Kegelschnitte, welche diese Curven in drei verschiedenen Puncten berühren.
}

(Von Herrn Otto Hesse, Prof. der Math. an der Universität zu Königsberg.)

(Fortsetzung der Abhandlungen No. 10. und No. 11. 28ten Bandes.)

1.

Wenn man durch $f$ eine homogene Function dritter Ordnung von den Variabeln $x_{1}, x_{2}, x_{3}$ bezeichnet, so wird die Determinante $\varphi$, gebildet aus den zweiten partiellen Differentialquotienten

$$
\begin{array}{ccc}
\frac{\partial^{2} f}{\partial x_{1}^{2}}, & \frac{\partial^{2} f}{\partial x_{1} \partial x_{2}}, & \frac{\partial^{2} f}{\partial x_{1} \partial x_{3}}, \\
\frac{\partial^{2} f}{\partial x_{2} \partial x_{1}}, & \frac{\partial^{2} f}{\partial x_{2}^{2}}, & \frac{\partial^{2} f}{\partial x_{2} \partial x_{3}}, \\
\frac{\partial^{2} f}{\partial x_{3} \partial x_{1}}, & \frac{\partial^{2} f}{\partial x_{3} \partial x_{2}}, & \frac{\partial^{2} f}{\partial x_{3}^{2}},
\end{array}
$$

wieder eine homogene Function der dritten Ordnung, welche ich in meiner Abhandlung „Über die Elimination der Variabeln aus drei algebraischen Gleichungen vom zweiten Grade mit zwei Variabeln (Bd. 28. S. 68)" mit dem Namen Determinante der Function $f$ bezeichnet habe. Dieser Bezeichnung werde ich 'mich auch bei der vorliegenden Untersuchung bedienen, welche als eine geometrische Interpretation und Erweiterung der in der citirten Schrift gewomnenen analytischen Resultate anzusehen ist.

Während nun die Bestimmung der Determinante einer gegebenen homogenen Function 3ten Grades von drei Variabeln nur die einfachsten analytischen Operationen erfordert, führt die umgekehrte Aufgabe: „Diejenige Function $\boldsymbol{F}$ zu bestimmen, deren Determinante eine gegebene homogene Function 3ter Ordnung von 3 Variabeln ist," auf eine Gleichung 3ten Grades. Denn ich habe in der citirten Abhandlung S. 89 bewiesen, dafs die gesuchte Function von der Form

$$
\boldsymbol{F}=\boldsymbol{d} \boldsymbol{f}+\delta \varphi
$$

sein mufs und dafs die Determinante $\Phi$ einer Function von dieser Form, wieder 
dieselbe Form

$$
\boldsymbol{\Phi}=\boldsymbol{D} \boldsymbol{f}+\Delta \varphi
$$

hat; wo $D$ und $\Delta$ homogene Functionen der Constanten $d$ und $\delta$ von der 3ten Ordnung sind. Auch die Bildung dieser Functionen habe ich angegeben *).

Wenn $\Phi$ gleich $f$ werden soll, so mufs

$$
\boldsymbol{D}=1 ; \quad \Delta=0
$$

sein. Dieses sind zwei Gleichungen dritten Grades in Rücksicht auf die zu bestimmenden Gröfsen $d$ und $\delta$, welche 9 Auflösungen zulassen. Da aber sowohl $\boldsymbol{D}$ als $\boldsymbol{A}$ homogene Functionen dritten Grades sind, in Rücksicht auf $d$ und $\delta$, so werden $\frac{D}{d^{3}}$ und $\frac{\Delta}{d^{3}}$ Functionen 3ten Grades der einen Variablen $\frac{\delta}{d}$ sein. Führt man diese statt $\delta$ als Unbekannte ein, so finden sich die beiden Unbekannten $d$ und $\frac{\delta}{d}$ aus den beiden Gleichungen

$$
d^{3}=\frac{1}{\frac{D}{d^{3}}} \text { und } \frac{\Delta}{d^{3}}=0 .
$$

Die zweite dieser Gleichungen giebt drei Werthe der zweiten Unbekannten. Setzt man diese Werthe in den Theil rechts der ersten Gleichung, so erhält man für jeden Werth der zweiten Unbekannten drei Werthe der

*) Die Bildung der Functionen $\boldsymbol{D}$ und $\Delta$ ist S. 88 durch die Formeln (51.)

$$
\boldsymbol{R D}=\Phi\left(\frac{1}{\varrho} \cdot q\right) ; \quad R \Delta=\Phi(p)
$$

angedeutet. Die Gröfse $R$ zu bestimmen, dienen die Gleichungen (32*. S.83), in welchen die Gröfsen $a$ und $b$ die Coüfficienten der Potenzen und Producte der Variabeln in den Functionen $f$ und $\varphi$, also bekannte Gröfsen bedeuten. Löset man diese, in Rücksicht auf die 6 Producte $x_{1} x_{1}, x_{2} x_{2}, x_{3} x_{3}, x_{2} x_{3}, x_{3} x_{1}, x_{1} x_{2}$ lineären Gleichungen auf, so als ob die 6 Producte die Unbekannten wăren: so erhält man, wie ich nachgewiesen habe, Gleichungen von der Form (33*), in welchen $\boldsymbol{R}$ den gemeinsamen Nenner der Unbekannten bedeutet. Sowohl dieser Nenner, als die Coëfficienten der 6 Gröfsen $f_{1}, f_{2}, f_{3}, \varphi_{1}, \varphi_{2}, \varphi_{3}$, welche mit $\frac{1}{\varrho} \cdot \eta$ und $p$ bezeichnet worden sind, werden durch die Auflösung bekannt. Bildet man endlich die Determinante $\Phi$ der Function $\boldsymbol{F}$ und setzt in derselben für alle Producte $x_{\psi} x_{\lambda} x_{\mu}$ entweder $\frac{1}{\varrho} \cdot q_{x, \lambda, \mu}$ oder $p_{x, \lambda, \mu}$, so erhält man im ersten Falle den mit $\Phi\left(\frac{1}{\varrho} q\right)$ bezeichneten Ausdruck, im zweiten Fall den Ausdruck $\Phi(p)$. Diese Bestimmung der Functionen $D$ und $\Delta$ läfst in der That nichts weiter als etwa gröfsere Einfachheiten zu wünschen übrig. Da gleichwohl Herr Cayley (Crelle's Journ. Bd. 29. pag. 55) behauptet, dafs ich die Form der Functionen $\boldsymbol{D}$ und $\Delta$ nicht angegeben habe, so sehe ich, dafs meine Darstellung einen Zweifel hat aufkommen lassen, den ich jetzt beseitigt zu haben glaube. 
ersten, welche sich nur durch einen Factor, gleich der dritten Wurzel der Einheit, von einander unterscheiden. Man findet also 9 Functionen $\boldsymbol{F}$, deren Determinanten die gegebene Function $f$ sind, wenn man die 9 Werthenpaare der Unbekannten in die Gleichung

$$
\boldsymbol{F}=d\left(r+\frac{\delta}{d} \varphi\right)
$$

setzl:

Von diesen 9 Functionen $\boldsymbol{F}$ sind aber nur die drei, welche den drei Wurzeln der cubischen Gleichung $\frac{\Delta}{d^{3}}=0$ entsprechen, wesentlich von einander verschieden. Deṇn es ergeben sich aus ihnen die 6 andern durch Multiplication mit den dritten Wurzeln der Einheit. Wenn im Folgenden von den drei verschiedenen Functionen $\boldsymbol{F}$ die Rede sein wird, so sollen darunter nur die drei ersten verstanden werden.

2.

Wenn $f$ eine der drei Functionen bedeutet, deren Determinante gleich einer beliebigen gegebenen homogenen Function $\varphi$ dritter Ordnung von den drei Variabeln $x_{1}, x_{2}, x_{3}$ ist und man setzt der Kürze wegen:

$$
\begin{array}{llrl}
\frac{\partial^{2} f}{\partial x_{1}^{2}}=u_{1,1} ; & \frac{\partial^{2} f}{\partial x_{2}^{2}}=u_{2,2} ; & & \frac{\partial^{2} f}{\partial x_{3}^{2}}=u_{3,3}, \\
\frac{\partial^{2} f}{\partial x_{2} \partial x_{3}}=u_{2,3}=u_{3,2} ; & \frac{\partial^{2} f}{\partial x_{3} \partial x_{1}}=u_{3,1}=u_{1,3} ; & \frac{\partial^{2} f}{\partial x_{1} \partial x_{2}}=u_{1,2}=u_{2,1},
\end{array}
$$

so erhält man die Gleichung $\varphi=0$, wenn man $\boldsymbol{X}_{1}, \boldsymbol{X}_{2}, \boldsymbol{X}_{3}$ zwischen folgenden drei Gleichungen eliminirt:

$$
\text { 1. } \quad\left\{\begin{array}{l}
\boldsymbol{X}_{1} u_{1,1}+X_{2} u_{1,2}+X_{3} u_{1,3}=0, \\
\boldsymbol{X}_{1} u_{2,1}+X_{2} u_{2,2}+X_{3} u_{2,3}=0, \\
\boldsymbol{X}_{1} u_{3,1}+X_{2} u_{3,2}+X_{3} u_{3,3}=0 .
\end{array}\right.
$$

Diese Gleichung $\varphi=0$ ist unter der Voraussetzung, dafs $\frac{x_{1}}{x_{3}}, \frac{x_{2}}{x_{3}}$, oder wie ich mich kürzer ausdrücken werde, $x_{1}, x_{2}, x_{3}$ die Coordinaten eines variabeln Punctes $p$ bezeichnen, der analytische Ausdruck einer beliebigen Curve dritter Ordnung. Diese Curve wird der Gegenstand der folgenden Untersuchung sein. Den Coordinaten $x_{1}, x_{2}, x_{3}$ jedes Punctes dieser Curve entspricht ein System Werthe $\boldsymbol{X}_{1}, \boldsymbol{X}_{2}, \boldsymbol{X}_{3}$, welches den Gleichungen (1.) genügt. Betrachtet man diese Werthe, in demselben Coordinatensystem, als die Coordinaten eines zweiten Punctes $\boldsymbol{P}$, so entspricht unter der Vermittelung der Gleichungen (1.) einem jeden Puncte $\boldsymbol{p}$ der Curve $\varphi=0$ ein anderer Punct $\boldsymbol{P}$ 
derselben Curve; und umgekehrt dem Puncte $\boldsymbol{P}$ der Punct $\boldsymbol{p}$. Denn man kann in den Gleichungen (1.) $x_{1}, x_{2}, x_{2}$ mit $X_{1}, X_{2}, X_{3}$ vertauschen, ohne die Gleichungen selbst zu ändern; weshalb auch das Resultat der Elimination von $x_{1}, x_{2}, x_{3}$, welches den geometrischen Ort des Punctes $\boldsymbol{P}$ giebt, aus der Gleichung $\varphi=0$ hervorgeht, wenn man $\boldsymbol{X}_{1}, \boldsymbol{X}_{2}, \boldsymbol{X}_{3}$ statt $x_{1}, x_{2}, x_{3}$ setzt.

Die Gleichungen (1.) lassen eine leichte geometrische Deutung zu. Sie sind die Bedingungsgleichungen zwischen den Coordinaten der beiden Puncte $\boldsymbol{P}$ und $\boldsymbol{p}$, welche erfüllt werden müssen, wenn die genannten beiden Puncte harmonische Pole der drei Kegelschnitte

$$
\frac{\partial f}{\partial x_{1}}=0, \quad \frac{\partial f}{\partial x_{2}}=0, \quad \frac{\partial f}{\partial x_{3}}=0
$$

sind; oder was dasselbe ist, eines jeden Kegelschnitts aus dem durch die Gleichung

$$
\lambda_{1} \frac{\partial f}{\partial x_{1}}+\lambda_{2} \frac{\partial f}{\partial x_{2}}+\lambda_{3} \frac{\partial f}{\partial x_{3}}=0
$$

dargestellten Systeme von Kegelschnitten. Die Curve dritter Ordnung $\varphi=0$ ist der geometrische Ort dieser Pole. (Harmonische Pole eines Kegelschnitts nennt man bekanntlich jedes Punctenpaar, von der Eigenschaft, dafs die durch sie gelegte gerade Linie den Kegelschnitt in einem zweiten Punctenpaar schneidet, welches zu dem ersten harmonisch ist.) Construirt man also ein Paar Puncte, welche harmonische Pole sind, zugleich für alle drei Kegelschnitte, oder für das ganze System von Kegelschnitten: so sind dieselben zwei unter der Vermittelung der Gleichungen (1.) einander entsprechende Puncte $\boldsymbol{P}$ und $\boldsymbol{p}$ und liegen beide in der betrachteten Curve dritter Ordnung $\varphi=0$.

Wenn zwei solcher Punctenpaare gegeben sind, so findet man ein drittes Paar durch Anwendung des folgenden Lehrsatzes, dessen Beweis ich in Bd. 20. S. 301 dieses Journals gegeben habe, nämlich: Wenn die Endpuncte zweier Diagonalen eines vollständigen Vierecks zwei Paare harmonischer Pole eines Kegelschnitts sind, so sind auch die Endpuncte der dritten Diagonale harmonische Pole desselben Kegelschnitls.

Der analytische Beweis dieses Satzes ergiebt sich aus den Bedingungsgleichungen zwischen den Coordinaten der 6 Puncte, welche die Diagonalen eines vollständigen Vierecks begrenzen. Wenn man nämlich durch $x_{1}, x_{2}, x_{3}$ und $X_{1}, X_{2}, X_{3}$ die Coordinaten der Endpuncte $p, P$ der ersten, durch $x_{1}{ }^{\prime}, x_{2}{ }^{\prime}, x_{3}{ }^{\prime}$ $\boldsymbol{X}_{1}^{\prime}, \boldsymbol{X}_{2}^{\prime}, \boldsymbol{X}_{3}^{\prime}$ die Endpuncte $\boldsymbol{p}^{\prime}, \boldsymbol{P}^{\prime}$ der zweiten Diagonale, endlich durch $x_{1}^{\prime \prime}, x_{2}^{\prime \prime}, x_{3}^{\prime \prime}$ und $X_{1}^{\prime \prime}, X_{2}^{\prime \prime}, X_{3}^{\prime \prime}$ die Endpuncte $\boldsymbol{p}^{\prime \prime}, \boldsymbol{P}^{\prime \prime}$ der dritten Diagonale 
bezeichnet, so sind die erwähnten Bedingungsgleichungen:

$$
\begin{aligned}
& A x_{1} X_{1}+A^{\prime} x_{1}^{\prime} X_{1}^{\prime}+A^{\prime \prime} x_{1}^{\prime \prime} X_{1}^{\prime \prime}=0 \text {, } \\
& \boldsymbol{A} x_{2} \boldsymbol{X}_{2}+\boldsymbol{A}^{\prime} \boldsymbol{x}_{2}{ }^{\prime} \boldsymbol{X}_{2}^{\prime}+\boldsymbol{A}^{\prime \prime} \boldsymbol{x}_{2}^{\prime \prime} \boldsymbol{X}_{2}^{\prime \prime}=0 \text {, } \\
& \boldsymbol{A} x_{3} \boldsymbol{X}_{3}+A^{\prime} x_{3}^{\prime} \boldsymbol{X}_{3}^{\prime}+A^{\prime \prime} x_{3}^{\prime \prime} \boldsymbol{X}_{3}^{\prime \prime}=0 \\
& \boldsymbol{A}\left(\boldsymbol{x}_{2} \boldsymbol{X}_{3}+\boldsymbol{x}_{3} \boldsymbol{X}_{2}\right)+\boldsymbol{A}^{\prime}\left(\boldsymbol{x}_{2}{ }^{\prime} \boldsymbol{X}_{3}^{\prime}+\boldsymbol{x}_{3}{ }^{\prime} \boldsymbol{X}_{2}^{\prime}\right)+\boldsymbol{A}^{\prime \prime}\left(\boldsymbol{x}_{2}{ }^{\prime \prime} \boldsymbol{X}_{3}^{\prime \prime}+\boldsymbol{x}_{3}{ }^{\prime \prime} \boldsymbol{X}_{2}^{\prime \prime}\right)=0, \\
& \boldsymbol{A}\left(x_{3} \boldsymbol{X}_{1}+x_{1} \boldsymbol{X}_{3}\right)+\boldsymbol{A}^{\prime}\left(\boldsymbol{x}_{3}{ }^{\prime} \boldsymbol{X}_{1}^{\prime}+x_{1}{ }^{\prime} \boldsymbol{X}_{3}^{\prime}\right)+A^{\prime \prime}\left(x_{3}^{\prime \prime} \boldsymbol{X}_{1}^{\prime \prime}+x_{1}{ }^{\prime \prime} \boldsymbol{X}_{3}^{\prime \prime}\right)=0, \\
& \boldsymbol{A}\left(x_{1} \boldsymbol{X}_{2}+x_{2} \boldsymbol{X}_{1}\right)+\boldsymbol{A}^{\prime}\left(\boldsymbol{x}_{1}{ }^{\prime} \boldsymbol{X}_{2}{ }^{\prime}+\boldsymbol{x}_{2}{ }^{\prime} \boldsymbol{X}_{1}^{\prime}\right)+\boldsymbol{A}^{\prime \prime}\left(\boldsymbol{x}_{1}{ }^{\prime \prime} \boldsymbol{X}_{2}{ }^{\prime \prime}+\boldsymbol{x}_{2}{ }^{\prime \prime} \boldsymbol{X}_{1}^{\prime \prime}\right)=0,
\end{aligned}
$$

in welchen die Gröfsen $\boldsymbol{A}, \boldsymbol{A}^{\prime}, \boldsymbol{A}^{\prime \prime}$ unbestimmte Constanten bedeuten. Stellt man nun die Bedingungsgleichungen

$$
\begin{aligned}
& \alpha_{1,1} \dot{x} x_{1} X_{1}+\alpha_{2,2} x_{2} X_{2}+\alpha_{3,3} x_{3} X_{3}+\alpha_{2,3}\left(x_{2} X_{3}+x_{3} X_{2}\right)+\alpha_{3,1}\left(x_{3} X_{1}+x_{1} X_{3}\right) \\
& +\alpha_{1,2}\left(x_{1} \boldsymbol{X}_{2}+x_{2} \boldsymbol{X}_{1}\right)=\mathbf{0} \\
& \alpha_{1,1} x_{1}{ }^{\prime} \boldsymbol{X}_{1}{ }^{\prime}+\alpha_{2,2} x_{2}{ }^{\prime} \boldsymbol{X}_{2}^{\prime}+\alpha_{3,3} x_{3} \boldsymbol{X}_{3}^{\prime}+\alpha_{2,3}\left(x_{2}{ }^{\prime} \boldsymbol{X}_{3}{ }^{\prime}+x_{3}{ }^{\prime} \boldsymbol{X}_{2}^{\prime}\right)+\alpha_{3, \mathbf{1}}\left(\boldsymbol{x}_{3}{ }^{\prime} \boldsymbol{X}_{1}^{\prime}+\boldsymbol{x}_{\mathbf{1}}{ }^{\prime} \boldsymbol{X}_{3}{ }^{\prime}\right) \\
& +\alpha_{1,2}\left(x_{1}^{\prime} \boldsymbol{X}_{2}^{\prime}+x_{2}^{\prime} \boldsymbol{X}_{1}^{\prime}\right)=0 \\
& \alpha_{1,1} x_{1}{ }^{\prime \prime} \boldsymbol{X}_{1}{ }^{\prime \prime}+\alpha_{2,2} x_{2}{ }^{\prime \prime} \boldsymbol{X}_{2}{ }^{\prime \prime}+\alpha_{3,3} x_{3}{ }^{\prime \prime} \boldsymbol{X}_{3}{ }^{\prime \prime}+\alpha_{2,3}\left(x_{2}{ }^{\prime \prime} \boldsymbol{X}_{3}{ }^{\prime \prime}+x_{3}{ }^{\prime \prime} \boldsymbol{X}_{2}{ }^{\prime \prime}\right)+\alpha_{3,1}\left(x_{3}{ }^{\prime \prime} \boldsymbol{X}_{1}{ }^{\prime \prime}+x_{1}{ }^{\prime \prime} \boldsymbol{X}_{3}{ }^{\prime \prime}\right) \\
& +\alpha_{1,2}\left(x_{1}{ }^{\prime \prime} \boldsymbol{X}_{2}^{\prime \prime}+x_{2}{ }^{\prime \prime} \boldsymbol{X}_{1}^{\prime \prime}\right)=0
\end{aligned}
$$

auf, welche erfüllt werden müssen, wenn die drei Punclenpaare harmonische Pole eines Kegelschnitts

$$
\alpha_{1,1} x_{1}^{2}+\alpha_{2,2} x_{2}^{2}+\alpha_{3,3} x_{3}^{2}+2 \alpha_{2,3} x_{2} x_{3}+2 \alpha_{3,1} x_{3} x_{1}+2 \alpha_{1,2} x_{1} x_{2}=0
$$

sein sollen, so wird man wahrnehmen, dafs sich die letzte Bedingungsgleichung mit Hülfe der beiden ersten und der vorhergehenden Systeme von 6 Gleichungen findet, wenn man die Gleichungen der Reihe nach mit $\alpha_{1,1}, \alpha_{2,2}, \alpha_{3,3}, \alpha_{2,3}, \alpha_{3,1}$, $\alpha_{1,2}$ multiplicirt und die Producte addirt.

Vermöge des eben bewiesenen Satzes erhält man nun aus zwei Punctenpaaren $\boldsymbol{P}, \boldsymbol{p} ; \boldsymbol{P}^{\prime}, \boldsymbol{p}^{\prime}$, deren Coordinaten den Gleichungen (1.) genügen, ein drittes Paar, wenn man der Curve dritter Ordnung ein Viereck einschreibt, dessen Diagonalen $\boldsymbol{P}_{\boldsymbol{p}}$ und $\boldsymbol{P}^{\prime} \boldsymbol{p}^{\prime}$ sind, dieses Viereck vervollständigt und die Endpuncte der dritten Diagonale nimmt. Dieses läfst sich auch als Lehrsatz wie folgt aussprechen: Wenn drei Puncte $\boldsymbol{P}, \boldsymbol{P}^{\prime}, \boldsymbol{P}^{\prime \prime}$ der Curve dritter Ordnung $p=0$ auf einer geraden Linie liegen, so bilden die ihnen unler der Vermittelung der Gleichungen (1.) entsprechenden Puncte $p, p^{\prime}, p^{\prime \prime}$ die Ecken eines Dreiecks, dessen Seiten $p^{\prime} p^{\prime \prime}, p^{\prime \prime} p, p p^{\prime}$ respective durch die Puncte $\boldsymbol{P}, \boldsymbol{P}^{\prime}, \boldsymbol{P}^{\prime \prime}$ gehen.

Es ergiebt sich hieraus zugleich eine leichte Construction des, einem beliebigen Puncte $\boldsymbol{P}$ der Curve entsprechenden Punctes $p$; wenn ein solches Punctenpaar $\boldsymbol{P}^{\prime}, \boldsymbol{p}^{\prime}$ gegeben ist. Denn verbindet man die Punctg $\boldsymbol{P}, \boldsymbol{P}^{\prime}$ durch 
eine gerade Linie, so schneidet dieselbe die Curve in einem Puncte $\boldsymbol{P}^{\prime \prime}$. Verbindet man diesen mit dem Puncte $\boldsymbol{p}^{\prime}$ durch eine zweite gerade Linie, so schneidet letztere die Curve in dem gesuchten Puncte $p$. Man erhält aber auch denselben Punct $\boldsymbol{p}$, wenn man den Schnittpunct $\boldsymbol{p}^{\prime \prime}$ der geraden Linie $\boldsymbol{P}_{\boldsymbol{p}}{ }^{\prime}$ und der Curve, mit dem Puncte $\boldsymbol{P}^{\prime}$ durch eine gerade Linie verbindet. Denn diese geht ebenfalls durch den Punct $p$.

Um zu jedem Puncte $\boldsymbol{P}$ den unter Vermittelung der Gleichungen (1.) entsprechenden Punct $\boldsymbol{p}$ construiren zu können, wenn nichts weiter als die Curve $\varphi=0$ selbst gegeben ist, bleibt noch übrig, die Construction eines Punctenpaares $\boldsymbol{P}^{\prime}, \boldsymbol{p}^{\prime}$ zu finden. Zu diesem Zwecke stellen wir uns den Punct $\boldsymbol{P}$ dem Puncte $\boldsymbol{P}^{\prime}$ so nahe gerückt vor, dafs beide zusammenfallen. Die gerade Linie $\boldsymbol{P \boldsymbol { P } ^ { \prime }}$ wird in diesem Falle zur Tangente der Curve. Die ihnen entsprechenden Puncte $\boldsymbol{p}, \boldsymbol{p}^{\prime}$ fallen ebenfalls zusammen. Zieht man nun von dem Schnittpuncte $\boldsymbol{P}^{\prime \prime}$ der Tangente $\boldsymbol{P} \boldsymbol{P}^{\prime}$ und der Curve die gerade Linie $\boldsymbol{P}^{\prime \prime} \boldsymbol{p}^{\prime}$, welche, wie wir gesehen haben, durch $p$ geht, so wird, weil $p$ und $p^{\prime}$ zusammenfallen, die gerade Linie $\boldsymbol{P}^{\prime \prime} \boldsymbol{p}^{\prime}$ eine Tangente im Puncte $\boldsymbol{p}$ werden. Demnach kann der, einem beliebigen Puncte $\boldsymbol{P}^{\prime}$ unter Vermittelung der Gleichungen (1.) entsprechende Punct $\boldsymbol{p}^{\prime}$ auf folgende Weise construirt werden. Man ziehe die Tangente in dem Puncte $\boldsymbol{P}^{\prime}$. Von dem Schnittpuncte $\boldsymbol{P}^{\prime \prime}$ derselben mit der Curve ziehe man eine andere Tangente. Der Berührungspunct dieser zweiten Tangente wird der gesuchte Punct $\boldsymbol{p}^{\prime}$ sein. Nun lassen sich aber von einem Puncte $\boldsymbol{P}^{\prime \prime}$ der Curve, wenn man die Tangente in diesem Puncte ausnimmt, 4 Tangenten an die Curve ziehen, von denen die eine die Tangente im Puncte $\boldsymbol{P}^{\prime}$ ist. Von den Berührungspuncten der drei andern wird demnach jeder dem Puncle $\boldsymbol{P}^{\prime}$ entsprechen. Und in der That giebt es auch drei, einem gegebenen Puncte $\boldsymbol{P}^{\prime}$ der Curve unter Vermittelung der Gleichungen (1.) entsprechende Puncte $\boldsymbol{p}^{\prime}$. Denn da in die Gleichungen (1.) die Coëfficienten aus der Fuuction $\boldsymbol{f}$ eingehen, aber, wie man gesehen hat, drei verschiedene Functionen $f$ existiren: so vereinigt auch das System Gleichungen (1.) drei verschiedene, den drei Functionen $f$ entsprechende Systeme von Gleichungen, und in jedem derselben entspricht ein - und demselben Puncte $\boldsymbol{P}$ ein anderer Punct $\boldsymbol{p}$. Die eben angegebene Construction läfst sich in Form eines Lehrsatzes wie folgt ausdrücken: Wenn man von cinesn beliebigen Puncte der Curve dritter Ordnung $\varphi=0$ die 4 Tangenten an die Curve zieht (die Tangente in dem beliebigen Puncte nicht mitgerechnet): so entsprechen einem jeden Tangi- 
rungspuncte die drei übrigen in den drei verschiedenen, durch die Gleichungen (1.) analy/isch ausgedrückten Systemen. Um diese drei Systeme sich entsprechender Puncte auch geometrisch zu sondern, ohne dazu der drei in (1.) enthaltenen Systeme von Gleichungen zu bedürfen, dient die vorangeschickte Construction des dem Puncte $\boldsymbol{P}$ entsprechenden Punctes $\boldsymbol{p}$, wenn ein solches Punctenpaar $\boldsymbol{P}^{\prime}, \boldsymbol{p}^{\prime}$ gegeben ist. Denn diese Construction giebt, wenn man den Punct $\boldsymbol{P}$ die ganze Curve durchlaufen läfst, alle möglichen Punctenpaare in demselben Systeme, wie das gegebene, während der zuletzt angeführte Satz drei, den drei verschiedenen Systemen zugehörende Punctenpaare construiren lehrt.

Um den zuletzt angeführten Lehrsatz zu vervollständigen, betrachten wir die 4 Tangirungspuncte $p, p^{\prime}, p^{\prime \prime}, p^{\prime \prime \prime}$ der von einem beliebigen Puncte 0 an die Curve dritter Ordnung gezogenen Tangenten. Von diesen 4 Puncten werden, wie wir bemerkt haben, die Punctenpare $p, p^{\prime} ; p, p^{\prime \prime} ; p, p^{\prime \prime \prime}$ den drei verschiedenen Systemen angehören, weil in einem jeden Systeme einem und demselben Puncte nur ein einziger entspricht. Das Punctenpaar $\boldsymbol{p}^{\prime}, \boldsymbol{p}^{\prime \prime}$, welches nach dem oben ausgesprochenen Lehrsatze einem von den drei Systemen angehört, kann nicht demjenigen Systeme eigen sein, welchem das erste Punctenpaar $\boldsymbol{p}, \boldsymbol{p}^{\prime}$ angehört. Denn wäre dieses der Fall, so müfste, wie aus dem Vorhergehenden erhellet, die Tangente im Puncte $p^{\prime}$ die gerade Linie $p p^{\prime \prime}$ in einem Puncte der Curve treffen, und dieser könnte nur der Punct 0 sein; was nicht möglich ist. Eben so wenig können die Punctenpaare $p^{\prime}, p^{\prime \prime}$ und $p, p^{\prime \prime}$ einem und demselben Systeme angehören. Also müssen es die Punctenpaare $p^{\prime}, p^{\prime \prime}$ und $p, p^{\prime \prime \prime}$ sein. Einem andern Systeme gehören nur die Punctenpaare $p, p^{\prime}$ und $p^{\prime \prime}, p^{\prime \prime \prime}$, und dem letzten Systeme gehören die Punctenpaare $p, p^{\prime \prime}$ und $p^{\prime}, p^{\prime \prime \prime}$ an. Diese Bemerkungen fassen wir in folgenden Satz zusammen: Wenn man von einem beliebigen Puncte der Curve dritter Ordnung $\varphi=0$ die 4 Tangenten an die Curve zieht, so lassen sich die 4 Thangirungspuncte als die Ecken von 3 versckiedenen Vierecken betrachten. Die gegenüberliegenden Ecken eines beliebigen dieser Vierecke sind zwei, demselben Systeme zugehörige Punctenpaare, und die Systeme, welche auf diese Weise den 3 verschiedenen Vierecken entsprechen, sind verschieden.

Man hat im Vorhergehenden gesehen, wie zwei Punctenpaare $\boldsymbol{P}, \boldsymbol{p} ; \boldsymbol{P}^{\prime}, \boldsymbol{p}^{\prime}$ desselben Systems ein durch dieselben gegebenes drittes Paar $\boldsymbol{P}^{\prime \prime}, \boldsymbol{p}^{\prime \prime}$ bestimmen, welches eben demselben Systeme angehört. Nunmehr will ich nachweisen, wie zwei Punctenpaare $\boldsymbol{P}, \boldsymbol{p} ; \boldsymbol{Q}, q$, aus zwei verschiedenen Systemen, ein drittes 
Punctenpaar $\boldsymbol{R}, \boldsymbol{r}$ des letzten Systems bestimmen. $\mathrm{Zu}$ diesem Ende lege ich durch die Curve dritter Ordnung zwei beliebige gerade Linien, von denen die eine die Curve in den Puncten $\boldsymbol{P}, \boldsymbol{Q}, \boldsymbol{R}$, die andere in den Puncten $\boldsymbol{P}^{\prime}, \boldsymbol{Q}^{\prime}, \boldsymbol{R}^{\prime}$ treffen möge. Wenn nun die drei geraden Linien $\boldsymbol{P P}, \boldsymbol{Q Q} \boldsymbol{Q}^{\prime}, \boldsymbol{R} \boldsymbol{R}^{\prime}$ die Curve respective in den Puncten $\boldsymbol{P}^{\prime \prime}, \boldsymbol{Q}^{\prime \prime}, \boldsymbol{R}^{\prime \prime}$ schneiden, so liegen diese drei Puncte $\boldsymbol{P}^{\prime \prime}, \boldsymbol{Q}^{\prime \prime}, \boldsymbol{R}^{\prime \prime}$ (was sich in den Elementen der Geometrie bewiesen findet) in einer geraden Linie. Diese läfst sich auch, wenn man drei gerade Linien als eine Curve dritter Ordnung, und zwei gerade Linien als einen Kegelschnitt betrachtet (was bekanntlich erlaubt ist), wie folgt ausdrücken: Wenn von den 9 Schnittpuncten zweier Curven dritter Ordnung 6 Schnittpuncte auf einem Kegelschnitt liegen, so liegen die drei andern auf einer geraden Linie. Läfst man die gerade Linie $\boldsymbol{P}^{\prime} \boldsymbol{Q}^{\prime} \boldsymbol{R}^{\prime}$ der geraden Linie $\boldsymbol{P Q R}$ so nahe rücken, dafs beide zusammenfallen, so erhält man den, eben wie der vorhergehende, bekannten Lehrsatz: Die Tangenten der Curve dritter Ordnung an drei Puncten, welche in einer und derselben geraden Linie liegen, schneiden die Curve in drei Puncten, welche wieder in einer geraden Linie liegen. Man vergleiche "Analyse de transversales par Poncelet" im gegenwärtigen Journal Bd. 8. S. $129-136$, wo man die beiden letzten und andere aus ihnen gefolgerte, mit der vorliegenden Untersuchung im Zusammenhange stehende Sätze findet.

Wenn nun $\boldsymbol{P}, \boldsymbol{p}$ und $\boldsymbol{Q}, \boldsymbol{q}$ zwei Punctenpaare in verschiedenen Systemen sind, so trifft, wie oben bewiesen worden, das Tangentenpaar der Curve in den Puncten $\boldsymbol{P}, \boldsymbol{p}$ in einem und demselben Puncte $\boldsymbol{P}^{\prime \prime}$ der Curve zusammen, und das Tangentenpaar der Curve in den Puncten $Q, q$ schneidet die Curve in einem und demselben Puncte $\boldsymbol{Q}^{\prime \prime}$. Läfst man die geraden Linien $\boldsymbol{P Q}$ und $p q$ die Curve respective in den Puncten $R$ und $r$ schneiden, so wird die Tangente in $\boldsymbol{R}$, nach dem zuletzt genannten Lehrsatze, die Curve in einem Puncte $\boldsymbol{R}^{\prime \prime}$ schneiden, welcher mit den beiden Puncten $\boldsymbol{P}^{\prime \prime}$ und $\boldsymbol{Q}^{\prime \prime}$ in einer und derselben geraden Linie liegt. Aus demselben Grunde mufs aber auch die Tangente in $\boldsymbol{r}$ die Curve in $\boldsymbol{R}^{\prime \prime}$ treffen. Es ist mithin $\boldsymbol{R}, \boldsymbol{r}$ ein Punctenpaar aus einem der drei Systeme; und zwar aus dem dritten. Denn wenn dieses System dasselbe wäre, welchem das Punctenpaar $\boldsymbol{P}, \boldsymbol{p}$ und $\boldsymbol{Q}, \boldsymbol{q}$ zugehört, so müfsten die geraden Linien $\boldsymbol{P Q}$ und $p q$ in einem und demselben Puncte der Curve zusammenstofsen; in welchem Falle die Punctenpaare $\boldsymbol{P}, \boldsymbol{Q}$ und $p, q$ einem und demselben Systeme angehören würden; was gegen die Voraussetzung ist. Wir haben also folgenden Lehrsatz bewiesen: Wenn man 
10. Hesse, über Curven dritter Ordnung.

ein Punctenpaar aus einem der drei Systeme mit einem zweiten Punctenpaar aus einem anderen Systeme durch zwei gerade Linien verbindet, so schneiden diese die Curve dritter Ordnung in einem Punctenpare, welches dem dritten Systeme angehört.

Die bis hieher gemachten Bemerkungen werde ich nun, geordnet, mit verwandten Sätzen zusammenstellen, deren Beweise sich leicht aus den entwickelten Principien ergeben.

3.

1. Der geometrische Ort eines, dreien, beliebig gegebenen Kegelschnitten gemeinschaftlichen harmonischen Polenpaares ist eine Curve dritter Ordnung.

2. Jede gegebene Curve dritter Ordnung lä/st sich als der geometrische Ort eines, einem Systeme Kegelschnitle gemeinschaftlichen harmonischen Polenpaares betrachten.

3. Solcher Systeme Kegelschnilte giebt es für jede beliebige Curve dritter Ordnung im Allgemeinen drei; also auch drei verschiedene Systeme Polenpaare auf einer und derselben Curve dritter Ordnung.

4. Das Tangentenpaar in einem Polenpaar an die Curve dritter Ordnung schneidet die Curve in einem und dernselben Puncte. Und umgekehrt:

5. Jedes Tangentenpaar, von einem beliebigen Puncte der Curve dritter Ordnung an die Curve gezogen, berührt die Curve in einem Polenpaare.

Die Sätze (4. und 5.) sind unmittelbare Folgen aus den beiden folgenden allgemeinen Sätzen:

6. In jedem der Curve dritter Ordnung einbeschriebenen Viereck, dessen Diagonalen von zwei Polenpaaren desselben Systems begrenzt werden, schneiden die gegenüberliegenden Seiten des Vierecks die Curve in einem und demselben Puncte; und die beiden Puncte, in welchen zwei aufeinanderfolgende Seiten des Vierecks die Curve schneiden, bilden ein Polenpaar desselben Systems. Und umgekehrt:

7. Die Diagonalen eines jeden, einer Curve dritter Ordnung einbeschriebenen vollständigen Vierecks werden von Polenpaaren begrenst, welche einem und demselben Systeme angehören.

Aus den vorhergehenden Sätzen ergiebt sich eine allgemeine Construction der einer Curve dritter Ordnung einbeschriebenen vollstăndigen Vierecke, welche in Form eines Lehrsatzes also lautet: 
8. Wenn man irgend ein Polenpaar $\boldsymbol{P}^{\prime}, p^{\prime}$ einer Curve dritter Ordnung durch zwei gerade Linien mit einem beliebigen Puncte $\boldsymbol{P}^{\prime \prime}$ der Curve, und die Schnittpuncte $\boldsymbol{P}, \boldsymbol{p}$ dieser beiden geraden Linien und der Curve durch zuei neue gerade Linien mit dem Polenpaare $\boldsymbol{P}^{\prime}, p^{\prime}$ verbindet, so bilden die beiden Linienpanre ein der Curve einbeschriebenes vollständiges Viereck.

9. Man kann alle, einer Curve dritter Ordnung einbeschriebenen vollstündigen Vierecke in drei verschiedene Systeme vertheilen. Ein beliebiges vollständiges, der Curve einbeschriebenes Viereck gehört dem einen oder dem andern Systeme an, je nachdem die, eine beliebige Diagonale desselben begrenzenden Puncte, ein Polenpar des einen und des andern Systems sind.

10. Alle einer Curve dritter Orinung einbeschriebenen Dreiecke, deren Seiten die Curve in drei Puncten schneiden, welche in einer geraden Linie liegen, ordnen sich dreien Systemen solcher Dreiecke unter. Ein solches Dreieck gehört dem einen oder dem andern Systeme an, je nachdem eine Ecke und der Schnittpunct der gegenüberliegenden Seite des Dreiecks und der Curve ein Polenpaar aus dem einen oder dem andern. Systeme sind.

11. Es lassen sich einer Curve dritter Ordnung nur drei Dreiecke einbeschreiben, deren Seiten durch drei, auf der Curve gegebene, in einer geraden Linie liegende Puncte hindurchgehen; und diese Dreiecke gehören verschiedenen Systemen an.

12. Wenn man von einem beliebigen Puncte einer Curve dritter Ordnung die vier Tangenten an die Curve zieht, so ist ein beliebiger von den 4 Berührungspuncten der Pol zu den drei andern, aus verschiedenen Systemen genommen; und wenn man drei von den Berührungspuncten als die Ecken eines Dreiecks betruchtet, so werden die Seiten dieses Dreiecks von Polenpaaren aus verschiedenen Systemen begrenst.

13. Wenn von drei Puncten auf einer Curve dritter Ordnung ein Punct der Pol ist zu den beiden andern, in zwei verschiedenen Systemen, so bilden die beiden letzten ein Polenpaar aus dem dritten Systeme.

14. Wenn man ein Polenpaar auf einer Curve dritter Ordnung mit einem zweiten Polenpaire derselben Curve aus einem andern Systeme durch zwei gerade Linien verbindet, so schneiden diese Linien die Curve in einem Polenpaare des dritten Systems.

15. Wenn man von einem beliebigen Puncte $p$ einer Curve drilter Ordnung die eine Tangente an die Curve zieht, so trifft jedes durch die 
vier Berührungspuncte gelegte Linienpaar die Curve in einem und demselben Puncte, und von den drei Puncten, in welchen die drei Linienpaare, welche durch die vier Puncte gelegt werden können, die Curve schneiden, bilden je zwei Potenzen, welche verschiedenen Systemen angehören. Diese drei Puncte und der Punct $p$ sind die Berührungspuncte der vier von einem und demselben Puncte der Curve an die Curve gezogenen Tangenten.

16. Wenn man aus den drei Schnittpuncten einer beliebiegen geraden Linie und einer Curve dritter Ordnung die 12 Tangenten an die Curve zieht, so liegen von den 12 Berührungspuncten 16 mal drei Puncte in einer geruden Linie.

Um die Combinationen derjenigen Berührungspuncte aufzustellen, welche in einer geraden Linie liegen, bezeichne ich irgend drei von den 12 Berührungspuncten, die in einer geraden Linie liegen, durch $\alpha, \beta, \gamma$; die diesen entsprechenden Pole in dem ersten Systeme durch $\alpha_{1}, \beta_{1}, \gamma_{1}$, in dem zweiten Systeme durch $\alpha_{2}, \beta_{2}, \gamma_{2}$ und in dem letzten Systeme durch $\alpha_{3}, \beta_{3}, \gamma_{3}$. Alsdann giebt es folgende Combinationen der 9 letzten Puncte, welche in einer geraden Linie liegen:

$$
\begin{array}{lll}
\alpha_{1} \beta_{2} \gamma_{3}, & a_{2} \beta_{3} \gamma_{1}, & \alpha_{3} \beta_{1} \gamma_{2}, \\
\alpha_{1} \beta_{3} \gamma_{2}, & \alpha_{2} \beta_{1} \gamma_{3}, & \alpha_{3} \beta_{2} \gamma_{1} ;
\end{array}
$$

was durch den Lehrsatz (14.) bewiesen wird. Überdies giebt es noch folgende Combinationen der 12 Berührungspuncte, welche in gerader Linie liegen:

$$
\begin{array}{ccc}
\alpha \beta_{1} \gamma_{1}, & \beta \gamma_{1} \alpha_{1}, & \gamma \alpha_{1} \beta_{1}, \\
\alpha \beta_{2} \gamma_{2}, & \beta \gamma_{2} \alpha_{2}, & \gamma \alpha_{2} \beta_{2}, \\
\alpha \beta_{3} \gamma_{3}, & \beta \gamma_{3} \alpha_{3}, & \gamma \alpha_{3} \beta_{3}, \\
& \alpha \beta \gamma . &
\end{array}
$$

Dies folgt aus dem Lehrsatze (6.). Demnach sind die in dem Lehrsatze (11.) erwähnten, der Curve einbeschriebenen Dreiecke, deren Seiten durch die drei in gerader Linie und zugleich auf der Curve gelegene Puncte $\alpha, \beta, \gamma$ hindurchgehen, folgende drei :

$$
\alpha_{1} \beta_{1} \gamma_{1}, \quad \alpha_{2} \beta_{2} \gamma_{2}, \quad \alpha_{3} \beta_{3} \gamma_{3} .
$$

Die aufgestellten Combinationen zeigen, dafs es 8 Systeme von je 4 geraden Linien giebt, welche durch sämmtliche 12 Berührungspuncte hindurchgehen. Die 4 geraden Linien gehen nämlich durch die Puncte:

Crelle's Journal f. d. M. Bd. XXXVI. Heft 2. 


$\begin{array}{lllll}\text { 1. } & \alpha \beta \gamma, & \alpha_{1} \beta_{2} \gamma_{3}, & \alpha_{2} \beta_{3} \gamma_{1}, & \alpha_{3} \beta_{1} \gamma_{2}, \\ \text { 2. } & \alpha \beta_{1} \gamma_{1}, & \alpha_{2} \beta_{2} \gamma, & \alpha_{1} \beta_{3} \gamma_{2}, & \alpha_{3} \beta \gamma_{3}, \\ \text { 3. } & \alpha \beta_{2} \gamma_{2}, & \alpha_{3} \beta_{3} \gamma, & \alpha_{1} \beta \gamma_{1}, & \alpha_{2} \beta_{1} \gamma_{3}, \\ \text { 4. } & \alpha \beta_{3} \gamma_{3}, & \alpha_{1} \beta_{1} \gamma, & \alpha_{2} \beta \gamma_{2}, & \alpha_{3} \beta_{2} \gamma_{1}, \\ \text { 5. } & \alpha \beta \gamma, & \alpha_{1} \beta_{3} \gamma_{2}, & \alpha_{2} \beta_{1} \gamma_{3}, & \alpha_{3} \beta_{2} \gamma_{1}, \\ \text { 6. } & \alpha \beta_{1} \gamma_{1}, & \alpha_{1} \beta_{2} \gamma_{3}, & \alpha_{2} \beta \gamma_{2}, & \alpha_{3} \beta_{3} \gamma, \\ \text { 7. } & \alpha \beta_{2} \gamma_{2}, & \alpha_{1} \beta_{1} \gamma, & \alpha_{2} \beta_{3} \gamma_{1}, & \alpha_{3} \beta \gamma_{3}, \\ \text { 8. } & \alpha \beta_{3} \gamma_{3}, & \alpha_{1} \beta \gamma_{1}, & \alpha_{2} \beta_{2} \gamma, & \alpha_{3} \beta_{1} \gamma_{2} .\end{array}$

Hieraus ist ersichtlich, dafs die 12 Berührungspuncte auf einer Curve vierter Ordnung liegen. Wie die Gleichung dieser Curve gebildet werden kann, giebt folgender Lehrsatz an:

17. Wenn $v$ eine gegebene homogene Function dritten Grades der drei Variabeln $x_{1}, x_{2}, x_{3}$, also

$$
\text { I. } v=0
$$

die Gleichung einer gegebenen Curve dritter Ordnung und

$$
\text { II. } \quad a_{1} x_{1}+a_{2} x_{2}+a_{3} x_{3}=0
$$

die Gleichung einer gegebenen geraden Linie ist, und man bezeichnet durch $w$ die Determinante der Function $v$, gebildet aus den zweiten partiellen Differentialquotienten dieser Function, so ist

$$
\text { III. } \begin{gathered}
\boldsymbol{a}_{1}\left(\frac{\partial v}{\partial x_{2}} \frac{\partial w}{\partial x_{3}}-\frac{\partial v}{\partial x_{3}} \frac{\partial w}{\partial x_{2}}\right)+\boldsymbol{a}_{2}\left(\frac{\partial v}{\partial x_{3}} \frac{\partial w}{\partial x_{1}}-\frac{\partial v}{\partial x_{1}} \frac{\partial w}{\partial x_{3}}\right) \\
+\boldsymbol{a}_{3}\left(\frac{\partial v}{\partial x_{1}} \frac{\partial w}{\partial x_{2}}-\frac{\partial v}{\partial x_{2}} \frac{\partial w}{\partial x_{1}}\right)=0
\end{gathered}
$$

die Gleichung der Curve 4ter Ordnung, welche durch die Berührungspuncte der von den Schnittpuncten der gegebenen geraden Linie und der Curve dritter Ordnung an die letztere gezogenen Tangenten hindurchgeht.

Hieraus folgt, wenn man den Theil links der Gleichung (III.) der Kürze wegen durch $r$, und durch $b_{1}, b_{2}, b_{3}$ unbestimmte Coëfficienten bezeichnet, dafs:

IV. $v\left(b_{1} x_{1}+b_{2} x_{2}+b_{3} x_{3}\right)+r=0$

der allgemeine Ausdruck für die Curven 4ter Ordnung sein wird, welche durch die erwähnten 12 Berührungspuncte hindurchgehen; man wird die Coëfficienten $b_{1}, b_{2}, b_{3}$ in dieser Gleichung $8 \mathrm{mal}$ so bestimmen können, dafs der Theil links der Gleichung in 4 lineäre Factoren zerfällt.

Ich werde nun zeigen, wie der zuletzt aufgestellte Lehrsatz sich auf Curven nter Ordnung ausdehnen läfst, und in welchem Zusammenhange diese Ausdehnung des Satzes mit dem Problem der Doppeltangenten der Curven steht. 
Es sei $v=0$ die zwischen den Variabeln $x_{1}, x_{2}, x_{3}$ homogene Gleichung einer beliebigen gegebenen Curve $n$ ter Ordnung; $v_{1}, v_{2}, v_{3}$ seien die partiellen Differentialquotienten der Function $v$, nach den Variabeln genommen, und $\boldsymbol{X}_{1}, \boldsymbol{X}_{2}, \boldsymbol{X}_{3}$ die Coordinaten eines beliebig gegebenen Punctes $\boldsymbol{P}$ aufserhalb der Curve. Die $\boldsymbol{n}(\boldsymbol{n}-1)$ Berührungspuncte der vom Puncte $\boldsymbol{P}$ an die Curve gezogenen Tangenten werden bekanntlich durch den Schnitt der beiden Curven

IV. $a$. $v=0$ und $\quad v_{1} X_{1}+v_{2} X_{2}+v_{3} X_{3}=0$

bestimmt. Rückt der Punct $\boldsymbol{P}$ in die Curve $v=0$, so fallen mit ihm zwei Berührungspuncte zusammen und es lassen sich von diesem Puncte nur noch $n(n-1)-2$ Tangenten an die Curve ziehen, wenn man die Tangenten in dem Puncte $\boldsymbol{P}$ selbst ausschliefst. Um die Curve zu finden, in welcher die Berührungspuncte sämmtlicher $n^{2}(n-1)$ Tangenten liegen, welche von den Schnittpuncten einer durch die Gleichung:

$$
\text { V. } \quad a_{1} x_{1}+a_{2} x_{2}+a_{3} x_{3}=0
$$

gegebenen geraden Linie und der gegebenen Curve $v=0$ an die letztere gezogen werden können, bezeichne ich die Function, in welche $v$ übergeht, wenn man $\boldsymbol{X}_{1}, \boldsymbol{X}_{2}, \boldsymbol{X}_{3}$ statt $x_{1}, x_{2}, x_{3}$ setzt, durch $\boldsymbol{V}$, und durch $(\boldsymbol{V})$ den Ausdruck, in welchen $\boldsymbol{V}$ durch die Substitution von

VI. $\quad X_{1}=v_{2} a_{3}-v_{3} a_{2}, \quad X_{2}=v_{3} a_{1}-v_{1} a_{3}, \quad X_{3}=v_{1} a_{2}-v_{2} a_{1}$ übergeht. Die $n^{2}(n-1)$ Berührungspuncte stellen sich dann als die Schnittpuncte der beiden Curven

$$
\text { VII. } v=0 \text { und }(V)=0
$$

dar, von denen die erstere vom $n$ ten, die andere vom $n(n-1)$ ten Grade ist. Von diesen $n^{2}(n-1)$ Schnittpuncten fallen aber mit jedem Schnittpuncte der geraden Linie (V.) und der Curve $v=0$ zwei zusammen, so dafs $2 n$ Schnittpuncte als auf einer Curve 2ter Ordnung liegend, deren Gleichung $\left(a_{1} x_{1}+a_{2} x_{2}+a_{3} x_{3}\right)^{2}$ $=0$ ist, zu betrachten sind. Nun weifs man, dafs, wenn von den $\boldsymbol{n}(\boldsymbol{p}+\boldsymbol{q})$ Schnittpuncten zweier Curven vom $n$ ten und $p$ +qten Grade $n q$ Puncte auf einer Curve qten Grades liegen, die übrigen $n p$ Puncte auf einer Curve $p$ ten Grades liegen müssen. In dem vorliegenden Falle werden also die $n^{2}(n-1)-2 n$ Schnittpuncte der Curven (VII.), welche nicht auf der geraden Linie (V.) liegen, auf einer Curve $n(n-1)-2$ ter Ordnung liegen; woraus der Schlufs zu ziehen ist, dafs $(\boldsymbol{V})$ von der Form

$$
\text { VIII. } \quad(\boldsymbol{V})=\frac{\boldsymbol{P}_{n} \cdot v-\boldsymbol{Q}_{n}\left(a_{1} x_{1}+a_{2} x_{2}+a_{3} x_{3}\right)^{2}}{1.2 \ldots n}
$$

sein wird, wo $\boldsymbol{P}_{n}$ und $\boldsymbol{Q}_{n}$ homogene Functionen der Variabeln, respective von 
den Graden $n(n-2)$ und $n(n-1)-2$, bedeuten. Hiernach stellen sich die $n^{2}(n-1)-2 n$ Berührungspuncte, welche nicht in der geraden Linie (V.) liegen, als die Schniltpuncte der beiden Curven

$$
\text { IX. } v=0 \text { und } Q_{n}=0
$$

dar.

Die Richtigkeit dieses geometrisch abgeleiteten Resultats werde ich auch auf rein analytischem Wege nachweisen; bei welcher Gelegenheit sich zugleich die Bildungsweise der Functionen $\boldsymbol{P}_{n}$ und $\boldsymbol{Q}_{n}$ herausstellen wird. Zu diesem Ende bezeichne ich durch $[v]$ die Function, in welche $v$ übergeht, wenn $\operatorname{man} x_{1}+\lambda X_{1}, x_{2}+\lambda X_{2}, x_{3}+\lambda X_{3}$ statt $x_{1}, x_{2}, x_{3}$ setzt. Setzt man nun $\boldsymbol{X}_{2} \partial_{x_{1}}+\boldsymbol{X}_{2} \partial_{x_{2}}+\boldsymbol{X}_{3} \partial_{x_{3}}=\partial$, so erhält man

$$
\text { X. }[v]=(v)+\lambda(\partial v)+\frac{\lambda^{2}}{1.2}\left(\partial^{2} v\right)+\ldots \frac{\lambda^{n-1}}{1.2 \ldots .(n-1)}\left(\partial^{n-1} v\right)+\frac{\lambda^{n}}{1.2 \ldots . n}\left(\partial^{n} v\right) .
$$

In dieser Gleichung betrachte ich $\boldsymbol{X}_{1}, \boldsymbol{X}_{2}, \boldsymbol{X}_{3}$ als Functionen von $x_{1}, x_{2}, x_{3}$, welche durch die Gleichungen (VI.) gegeben sind. Unter dieser Annahme sind die Glieder der Reihe rechts von dem Gleichheitszeichen homogene Functionen von den Geraden:

$$
\begin{aligned}
& 0,1, \quad 2, \quad 3, \quad \ldots n \quad \text { in Beziehung auf } a_{1}, a_{2}, a_{3} \text {, } \\
& 1,2, \quad 3, \quad 4, \quad \ldots n+1 \text { in Beziehung auf die Coëfficienten in } v \text {, } \\
& n, 2 n-2,3 n-4,4 n-6, \ldots n(n-1) \text { in Beziehung auf } x_{1}, x_{2}, x_{3} \text {. }
\end{aligned}
$$

Man sieht leicht, dafs das letzte Glied der Reihe gleich ist $\lambda^{n}(\boldsymbol{V})$. Es bleibt also, wenn man der Kürze wegen $a_{1} x_{1}+a_{2} x_{2}+a_{3} x_{3}=a$ setzt, zu beweisen übrig, dafs das letzte Glied der Reihe von der Form

$$
\left(\partial^{n} \boldsymbol{v}\right)=\boldsymbol{P}_{n} \cdot \boldsymbol{v}-\boldsymbol{Q}_{n} \cdot \boldsymbol{a}^{2}
$$

sei. Von derselben Form sind aber auch alle übrigen Glieder der Reihe, so dafs

$$
\text { XI. }\left(\partial^{\mu} \boldsymbol{v}\right)=\boldsymbol{P}_{\mu} \cdot \boldsymbol{v}-\boldsymbol{Q}_{\mu} \cdot \boldsymbol{a}^{2}
$$

ist, wo $\boldsymbol{P}_{\mu}$ und $\boldsymbol{Q}_{\mu}$ homogene Functionen bedeuten, respective von den Graden:

$$
\begin{aligned}
& \mu \text { und } \mu-2 \text { in Beziehung auf } a_{1}, a_{2}, a_{3} \text {, } \\
& \mu \text { und } \mu+1 \text { in Beziehung auf die Coëfficienten in } v \text {, } \\
& \mu(n-2) \text { und }(\mu+1)(n-2) \text { in Beziehung auf } x_{1}, x_{2}, x_{3} .
\end{aligned}
$$

Dafs die beiden ersten Glieder der Reibe diese Form haben, ist einleuchtend. Denn selzt man $(\boldsymbol{v})=\boldsymbol{P} . \boldsymbol{v}-\boldsymbol{Q} \cdot \boldsymbol{a}^{2}$ und $(\partial \boldsymbol{v})=\boldsymbol{P}_{1} \cdot \boldsymbol{v}-\boldsymbol{Q}_{1} \cdot \boldsymbol{a}^{2}$, so erhält man, weil $(\partial v)$ identisch $=0$ ist,

XII. $\quad \boldsymbol{P}=1, \quad Q=0, \quad P_{1}=0, \quad Q_{1}=0$. 
Ich werde nun zeigen, wie sich jedes Glied der Reihe durch die beiden vorhergehenden Glieder ausdrücken läfst. Zu diesem Ende setze ich

XIII. $\begin{cases}\boldsymbol{Y}_{1}=\boldsymbol{X}_{1} \frac{\partial \boldsymbol{X}_{1}}{\partial x_{1}}+\boldsymbol{X}_{2} \frac{\partial \boldsymbol{X}_{1}}{\partial x_{2}}+\boldsymbol{X}_{3} \frac{\partial \boldsymbol{X}_{1}}{\partial x_{3}}, & \boldsymbol{Z}_{1}=\boldsymbol{Y}_{1} \frac{\partial \boldsymbol{X}_{1}}{\partial x_{1}}+\boldsymbol{Y}_{2} \frac{\partial \boldsymbol{X}_{1}}{\partial x_{2}}+\boldsymbol{Y}_{3} \frac{\partial \boldsymbol{X}_{1}}{\partial x_{3}}, \\ \boldsymbol{Y}_{2}=\boldsymbol{X}_{1} \frac{\partial \boldsymbol{X}_{2}}{\partial x_{1}}+\boldsymbol{X}_{2} \frac{\partial \boldsymbol{X}_{2}}{\partial x_{2}}+\boldsymbol{X}_{3} \frac{\partial \boldsymbol{X}_{2}}{\partial x_{3}}, & \boldsymbol{Z}_{2}=\boldsymbol{Y}_{1} \frac{\partial \boldsymbol{X}_{2}}{\partial x_{1}}+\boldsymbol{Y}_{2} \frac{\partial \boldsymbol{X}_{2}}{\partial x_{2}}+\boldsymbol{Y}_{3} \frac{\partial \boldsymbol{X}_{2}}{\partial x_{3}}, \\ \boldsymbol{Y}_{3}=\boldsymbol{X}_{1} \frac{\partial \boldsymbol{X}_{3}}{\partial x_{1}}+\boldsymbol{X}_{2} \frac{\partial \boldsymbol{X}_{3}}{\partial x_{2}}+\boldsymbol{X}_{3} \frac{\partial \boldsymbol{X}_{3}}{\partial x_{3}}, & \boldsymbol{Z}_{3}=\boldsymbol{Y}_{1} \frac{\partial \boldsymbol{X}_{3}}{\partial x_{1}}+\boldsymbol{Y}_{2} \frac{\partial \boldsymbol{X}_{3}}{\partial x_{2}}+\boldsymbol{Y}_{3} \frac{\partial \boldsymbol{X}_{3}}{\partial x_{3}}\end{cases}$

Ferner gebe ich den aus den zweiten partiellen Differentialquotienten $\boldsymbol{v}_{1,1}, \boldsymbol{v}_{2,2}$, $v_{3,3}, v_{2,3}, v_{3,1}, v_{1,2}$ der Function $v$ zusammengesetzten Ausdrücken der Kürze wegen folgende Bezeichnungen:

XIV. $\quad\left\{\begin{aligned} V_{2,2}=v_{3,3} v_{1,1}-v_{3,1}^{2}, & V_{3,1}=v_{2,3} v_{2,1}-v_{2,2} v_{3,1}, \\ V_{3,3}=v_{1,1} v_{2,2}-v_{1,2}^{2}, & V_{1,2}=v_{3,1} v_{3,2}-v_{3,3} v_{1,2}, \\ w & =v_{1,1} v_{2,2} v_{3,3}+2 v_{1,2} v_{1,3} v_{2,3}-v_{1,1} v_{2,3}^{2}-v_{2,2} v_{3,1}^{2}-v_{3,3} v_{1,2}^{2} \\ A & =V_{1,1} a_{1}^{2}+V_{2,2} a_{2}^{2}+V_{3,3} a_{3}^{2}+2 V_{2,3} a_{2} a_{3}+2 V_{3,1} v_{3} v_{1}+2 V_{1,2} v_{1} v_{2} .\end{aligned}\right.$

Alsdann stellen sich die Gröfsen $\boldsymbol{Y}$, wenn man die Werthe von $\boldsymbol{X}_{1}, \boldsymbol{X}_{2}, \boldsymbol{X}_{3}$ und $\frac{\partial X_{1}}{\partial x_{1}}, \frac{\partial X_{1}}{\partial x_{2}}, \ldots$ setzt, nach den nöthigen Reductionen wie folgt dar:

$$
\text { XV. }\left\{\begin{array}{l}
Y_{1}=\frac{1}{n-1}\left\{\frac{1}{2} a \cdot \frac{\partial \Delta}{\partial a_{1}}-x_{1} \Delta\right\} \\
Y_{2}=\frac{1}{n-1}\left\{\frac{1}{2} a \cdot \frac{\partial \Delta}{\partial a_{2}}-x_{2} \Delta\right\} \\
Y_{3}=\frac{1}{n-1}\left\{\frac{1}{2} a \cdot \frac{\partial \Delta}{\partial a_{3}}-x_{3} \Delta\right\}
\end{array}\right.
$$

woraus sich, wenn man erwägt, dafs

$$
\begin{aligned}
& \frac{\partial \Delta}{\partial a_{1}} \cdot \frac{\partial X_{1}}{\partial x_{1}}+\frac{\partial \Delta}{\partial a_{2}} \cdot \frac{\partial X_{1}}{\partial x_{2}}+\frac{\partial \Delta}{\partial a_{3}} \cdot \frac{\partial X_{1}}{\partial x_{3}}=0 \\
& \frac{\partial \Delta}{\partial a_{1}} \cdot \frac{\partial \boldsymbol{X}_{2}}{\partial x_{1}}+\frac{\partial \Delta}{\partial a_{2}} \cdot \frac{\partial \boldsymbol{X}_{2}}{\partial x_{2}}+\frac{\partial \Delta}{\partial a_{3}} \cdot \frac{\partial \boldsymbol{X}_{2}}{\partial x_{3}}=\mathbf{0} \\
& \frac{\partial \Delta}{\partial a_{1}} \cdot \frac{\partial \boldsymbol{X}_{3}}{\partial x_{1}}+\frac{\partial \Delta}{\partial a_{2}} \cdot \frac{\partial \boldsymbol{X}_{3}}{\partial x_{2}}+\frac{\partial \Delta}{\partial a_{3}} \cdot \frac{\partial \boldsymbol{X}_{3}}{\partial x_{3}}=\mathbf{0}
\end{aligned}
$$

ist, durch Substitution in (XIII.) folgende Werthe der Gröfsen $\boldsymbol{Z}$ ergeben:

$$
\text { XVI. } \quad \boldsymbol{Z}_{1}=-\Delta \boldsymbol{X}_{1}, \quad \boldsymbol{Z}_{2}=-\Delta \boldsymbol{X}_{2}, \quad \boldsymbol{Z}_{3}=-\Delta \boldsymbol{X}_{3} .
$$

Jedes Glied der Reihe [ $v]$ ist eine homogene Function der Variabeln $x_{1}, x_{2}, x_{3}$, und zugleich eine homogene Function der Gröfsen $\boldsymbol{X}_{1}, \boldsymbol{X}_{2}, \boldsymbol{X}_{3}$, welche wie- 
derum homogene Functionen der Variabeln $x_{1}, x_{2}, x_{3}$ sind. Ich werde nun die Differentiation nach den Variabeln $x_{1}, x_{2}, x_{3}$ mit $d$ bezeichnen, wenn sowohl die Gröfsen $x$, als die Gröfsen $\boldsymbol{X}$, als variabel betrachtet werden: dagegen mit $\partial$, wenn nur die eine als variabel, die andere als constant betrachtet wird. Mit dieser Bezeichnung ist:

$$
\begin{aligned}
& \frac{\partial\left(\partial^{\mu} v\right)}{\partial x_{1}}=\frac{d\left(\partial^{\mu} v\right)}{d x_{1}}-\left\{\frac{\partial\left(\partial^{\mu} v\right)}{\partial \boldsymbol{X}_{1}} \cdot \frac{\partial \boldsymbol{X}_{1}}{\partial x_{1}}+\frac{\partial\left(\partial^{\mu} v\right)}{\partial \boldsymbol{X}_{2}} \cdot \frac{\partial \boldsymbol{X}_{2}}{\partial x_{1}}+\frac{\partial\left(\partial^{\mu} v\right)}{\partial \boldsymbol{X}_{3}} \cdot \frac{\partial \boldsymbol{X}_{3}}{\partial x_{1}}\right\} \\
& \frac{\partial\left(\partial^{\mu} v\right)}{\partial x_{2}}=\frac{d\left(\partial^{\mu} v\right)}{d x_{2}}-\left\{\frac{\partial\left(\partial^{\mu} v\right)}{\partial \boldsymbol{X}_{1}} \cdot \frac{\partial \boldsymbol{X}_{1}}{\partial x_{2}}+\frac{\partial\left(\partial^{\mu} v\right)}{\partial \boldsymbol{X}_{2}} \cdot \frac{\partial \boldsymbol{X}_{2}}{\partial x_{2}}+\frac{\partial\left(\partial^{\mu} v\right)}{\partial \boldsymbol{X}_{3}} \cdot \frac{\partial \boldsymbol{X}_{3}}{\partial x_{2}}\right\} \\
& \frac{\partial\left(\partial^{\mu} v\right)}{\partial x_{3}}=\frac{d\left(\partial^{\mu} v\right)}{d x_{3}}-\left\{\frac{\partial\left(\partial^{\mu} v\right)}{\partial \boldsymbol{X}_{1}} \cdot \frac{\partial \boldsymbol{X}_{1}}{\partial x_{3}}+\frac{\partial\left(\partial^{\mu} v\right)}{\partial \boldsymbol{X}_{2}} \cdot \frac{\partial \boldsymbol{X}_{2}}{\partial x_{3}}+\frac{\partial\left(\partial^{\mu} v\right)}{\partial x_{3}} \cdot \frac{\partial \boldsymbol{X}_{3}}{\partial x_{3}}\right\}
\end{aligned}
$$

Es ist aber

$$
\frac{\partial\left(\partial^{\mu} v\right)}{\partial \boldsymbol{X}_{1}}=\mu \frac{\partial\left(\partial^{\mu} \hat{v}\right)}{\partial x_{1}}, \quad \frac{\partial\left(\partial^{\mu} \vec{v}\right)}{\partial \boldsymbol{X}_{2}}=\mu \frac{\partial\left(\partial^{\mu} \vec{v}\right)}{\partial x_{2}}, \quad \frac{\partial\left(\partial^{\mu} v\right)}{\partial \boldsymbol{X}_{3}}=\mu \frac{\partial\left(\partial^{\prime}{ }_{v}^{\prime}\right)}{\partial x_{3}} ;
$$

mit Hülfe welcher Gleichungen sich das vorige System also darstellt:

$$
\begin{aligned}
& \frac{\partial\left(\partial^{\mu} v\right)}{\partial x_{1}}=\frac{d\left(\partial^{\mu} v\right)}{d x_{1}}-\mu\left\{\frac{\partial\left(\partial^{\mu-1} v\right)}{\partial x_{1}} \cdot \frac{\partial \boldsymbol{X}_{1}}{\partial x_{1}}+\frac{\partial\left(\partial^{\mu-1} v\right)}{\partial x_{2}} \cdot \frac{\partial \boldsymbol{X}_{2}}{\partial x_{1}}+\frac{\partial\left(\partial^{\mu-1} v\right)}{\partial x_{3}} \cdot \frac{\partial \boldsymbol{X}_{3}}{\partial x_{1}}\right\}, \\
& \frac{\partial\left(\partial^{\mu} v\right)}{\partial x_{2}}=\frac{d\left(\partial^{\mu} v\right)}{d}-\mu\left\{\frac{\partial\left(\partial^{\mu-1} v\right)}{\partial x_{1}} \cdot \frac{\partial \boldsymbol{X}_{1}}{\partial x_{2}}+\frac{\partial\left(\partial^{\mu-1} v\right)}{\partial x_{2}} \cdot \frac{\partial \boldsymbol{X}_{2}}{\partial x_{2}}+\frac{\partial\left(\partial^{\mu-1} v\right)}{\partial x_{3}} \cdot \frac{\partial \boldsymbol{X}_{3}}{\partial x_{2}}\right\}, \\
& \frac{\partial\left(\partial^{\mu} v\right)}{\partial x_{3}}=\frac{d\left(\partial^{\mu} v\right)}{d x_{3}}-\mu\left\{\frac{\partial\left(\partial^{\mu-1} v\right)}{\partial x_{1}} \cdot \frac{\partial \boldsymbol{X}_{1}}{\partial x_{3}}+\frac{\partial\left(\partial^{\mu-1} v\right)}{\partial x_{2}} \cdot \frac{\partial \boldsymbol{X}_{2}}{\partial x_{3}}+\frac{\partial\left(\partial^{\mu-1} v\right)}{\partial x_{3}} \cdot \frac{\partial \boldsymbol{X}_{3}}{\partial x_{3}}\right\} .
\end{aligned}
$$

Multiplicirt man diese Gleichungen der Reihe nach mit $\boldsymbol{X}_{1}, \boldsymbol{X}_{2}, \boldsymbol{X}_{3}$, addirt die Producte und erwägt, dafs

$$
\left(\partial^{\mu+1} v\right)=\frac{\partial\left(\partial^{\mu} v\right)}{\partial x_{1}} \boldsymbol{X}_{1}+\frac{\partial\left(\partial^{\mu} v\right)}{\partial x_{2}} \boldsymbol{X}_{2}+\frac{\partial\left(\partial^{\mu} v\right)}{\partial x_{3}} \boldsymbol{X}_{3}
$$

ist, so erhält man, mit Rücksicht auf (XIII.),

$$
\begin{aligned}
\left(\partial^{\mu+1} v\right)= & \frac{d\left(\partial^{\mu} v\right)}{d} X_{1}+\frac{d\left(\partial^{\mu} v\right)}{d x_{2}} \boldsymbol{X}_{2}+\frac{d\left(\partial^{\mu} v\right)}{d x_{3}} \boldsymbol{X}_{3} \\
& -\mu\left\{\frac{\partial\left(\partial^{\mu-1} v\right)}{\partial x_{1}} \boldsymbol{Y}_{1}+\frac{\partial\left(\partial^{\mu-1} v\right)}{\partial x_{2}} \boldsymbol{Y}_{2}+\frac{\partial\left(\partial^{\mu-1} v\right)}{\partial x_{3}} \boldsymbol{Y}_{3}\right\}
\end{aligned}
$$

Setzt man in diese Gleichung diejenigen Werthe von $\frac{\partial\left(\partial^{\mu-1} v\right)}{\partial x_{1}}, \frac{\partial\left(\partial^{\mu-1} v\right)}{\partial x_{2}}, \frac{\partial\left(\partial^{\mu-1} v\right)}{\partial x_{3}}$, welche sich aus dem vorhergehenden Systeme von Gleichungen ergeben, wenn man in demselben $\mu-1$ statt $\mu$ setzt, so geht dieselbe, mit Rücksicht auf (XIII.) und (XIV.), in 
über. Da aber

$$
\begin{aligned}
& \left(\partial^{\mu+1} v\right)=\frac{d\left(\partial^{\mu} v\right)}{d x_{1}} X_{1}+\frac{d\left(\partial^{\mu} v\right)}{d x_{2}} X_{2}+\frac{d\left(\partial^{\mu} v\right)}{d} X_{3} X_{3} \\
& -\mu\left\{\frac{d\left(\partial^{\mu-1} v\right)}{d x_{1}} Y_{1}+\frac{d\left(\partial^{\mu-1} v\right)}{d x_{2}} Y_{2}+\frac{d\left(\partial^{\mu-1} v\right)}{d x_{3}} Y_{3}\right\} \\
& \left.-\mu(\mu-1) \frac{\partial\left(\partial^{\mu-2} v\right)}{\partial x_{1}} \boldsymbol{X}_{1}+\frac{\partial\left(\partial^{\mu-2} v\right)}{\partial x_{2}}+\boldsymbol{X}_{2} \frac{\partial\left(\partial^{\mu-2} v\right)}{\partial x_{3}} \underset{Z_{3}}{\boldsymbol{X}_{3}}\right\}
\end{aligned}
$$

$$
\begin{gathered}
\frac{\partial\left(\partial^{\mu-1} v\right)}{\partial \boldsymbol{X}_{1}}=(\mu-1) \frac{\partial\left(\partial^{\mu-2} v\right)}{\partial x_{1}}, \quad \frac{\partial\left(\partial^{\mu-1} v\right)}{\partial \boldsymbol{X}_{2}}=(\mu-1) \frac{\partial\left(\partial^{\mu-2} v\right)}{\partial x_{2}}, \\
\frac{\partial\left(\partial^{\mu-1} v\right)}{\partial \boldsymbol{X}_{3}}=(\mu-1) \frac{\partial\left(\partial^{\mu-2} v\right)}{\partial x_{3}} \\
\frac{\partial\left(\partial^{\mu-1} v\right)}{\partial \boldsymbol{X}_{1}} \boldsymbol{X}_{1}+\frac{\partial\left(\partial^{\mu-1} v\right)}{\partial \boldsymbol{X}_{2}} \boldsymbol{X}_{2}+\frac{\partial\left(\partial^{\mu-1} v\right)}{\partial \boldsymbol{X}_{3}} \boldsymbol{X}_{3}=(\mu-1)\left(\partial^{\mu-1} v\right)
\end{gathered}
$$

ist, so ergiebt sich

$$
\begin{aligned}
\left(\partial^{\mu+1} v\right)= & \frac{d\left(\partial^{\mu} v\right)}{d x_{1}} X_{1}+\frac{d\left(\partial^{\mu} v\right)}{d x_{2}} X_{2}+\frac{d\left(\partial^{\mu} v\right)}{d x_{3}} X_{3} \\
& -\mu\left\{\frac{d\left(\partial^{\mu-1} v\right)}{d x_{1}} Y_{1}+\frac{d\left(\partial^{\mu-1} v\right)}{d x_{2}} Y_{2}+\frac{d\left(\partial^{\mu-1} v\right)}{d x_{3}} Y_{3}\right\} \\
& -\mu(\mu-1) \Delta\left(\partial^{\mu-1} v\right) ;
\end{aligned}
$$

woraus endlich folgt, wenn man die Werthe von $\boldsymbol{Y}$ substituirt:

XVII.

$$
\begin{aligned}
\left(\partial^{\mu+1} v\right)= & \frac{d\left(\partial^{\mu} v\right)}{d x_{1}} X_{1}+\frac{d\left(\partial^{\mu} v\right)}{d x_{2}} X_{2}+\frac{d\left(\partial^{\mu} v\right)}{d x_{3}} X_{3} \\
& -\frac{\mu}{n-1} \cdot \frac{1}{2} a\left\{\frac{d\left(\partial^{\mu-1} v\right)}{d x_{1}} \cdot \frac{\partial \Delta}{\partial a_{1}}+\frac{d\left(\partial^{\mu-1} v\right)}{d x_{2}} \cdot \frac{\partial \Delta}{\partial a_{2}}+\frac{d\left(\partial^{\mu-1} v\right)}{d x_{3}} \cdot \frac{\partial \Delta}{\partial a_{3}}\right\} \\
& +\frac{\mu}{n-1}(n-\mu+1) \cdot \Delta \cdot\left(\partial^{\mu-1} v\right) .
\end{aligned}
$$

Dieses ist die gesuchte Gleichung, welche jedes Glied der Reihe [v] durch die beiden vorhergehenden ausdrückt. Es läfst sich aus ihr sogleich die Form des Ausdrucks $\left(\partial^{\mu+1} v\right)$ erkennen, wenn man berücksichtigt, dafs

$$
v_{1} \cdot \frac{\partial \Delta}{\partial a_{1}}+v_{2} \cdot \frac{\partial \Delta}{\partial a_{2}}+v_{3} \cdot \frac{\partial \Delta}{\partial a_{3}}=\frac{2}{n-1} \cdot a \cdot w
$$

ist. Wenn nämlich die Ausdrücke $\left(\partial^{\mu} v\right)$ und $\left(\partial^{\mu-1} v\right)$ von der Form (XI.) sind, so mufs auch $\left(\partial^{\mu+1} v\right)$ diese Form haben. Denn setzt man

$$
\left(\partial^{\mu} v\right)=\boldsymbol{P}_{\mu}^{\nu}-\boldsymbol{Q}_{\mu} \cdot a^{2} \text { und }\left(\partial^{\mu-1} v\right)=\boldsymbol{P}_{\mu-1} v_{\mu-1} \boldsymbol{Q}_{\mu}
$$

so erhält man aus (XVII.)

XVIII. $\quad\left(\partial^{\mu+1} v\right)=\boldsymbol{P}_{\mu+1} \cdot v-\boldsymbol{Q}_{\mu+1} \cdot a^{2}$,

wo die Werthe von $\boldsymbol{P}_{\mu+1}$ und $\boldsymbol{Q}_{\mu+1}$ folgende sind: 
XIX.

$$
\left\{\begin{aligned}
\boldsymbol{P}_{\mu+1}= & \left(\frac{d \boldsymbol{P}_{\mu}}{d x_{1}} \boldsymbol{X}_{1}+\frac{d \boldsymbol{P}_{\mu}}{d x_{2}} \boldsymbol{X}_{2}+\frac{d \boldsymbol{P}_{\mu}}{d x_{3}} \boldsymbol{X}_{3}\right) \\
& -\frac{\mu}{n-1} \cdot \frac{1}{2} a\left(\frac{d \boldsymbol{P}_{\mu-1}}{d x_{1}} \cdot \frac{\partial \Delta}{\partial a_{1}}+\frac{d \boldsymbol{P}_{\mu-1}}{d x_{2}} \cdot \frac{\partial \Delta}{\partial a_{2}}+\frac{d \boldsymbol{P}_{\mu-1}}{d x_{3}} \cdot \frac{\partial \Delta}{\partial a_{3}}\right) \\
& +\frac{\mu(n-\mu+1)}{n-1} \cdot \Delta \cdot \boldsymbol{P}_{\mu-1}, \\
\boldsymbol{Q}_{\mu+1}= & \left(\frac{d Q_{\mu}}{d x_{1}} \boldsymbol{X}_{1}+\frac{d Q_{\mu}}{d x_{2}} \boldsymbol{X}_{2}+\frac{d Q_{\mu}}{d x_{3}} \boldsymbol{X}_{3}\right) \\
& -\frac{\mu}{n-1} \cdot \frac{1}{2} a\left(\frac{d Q_{\mu-1}}{d x_{1}} \cdot \frac{\partial \Delta}{\partial a_{1}}+\frac{d Q_{\mu-1}}{d x_{2}} \cdot \frac{\partial \Delta}{\partial a_{2}}+\frac{d Q_{\mu-1}}{d x_{3}} \cdot \frac{\partial d}{\partial a_{3}}\right) \\
& +\frac{\mu}{(n-1)^{2}} w \cdot \boldsymbol{P}_{\mu-1}+\frac{\mu(n-\mu-1)}{n-1} \cdot \Delta \cdot Q_{\mu-1} .
\end{aligned}\right.
$$

Setzt man in diesen Gleichungen für $\mu$ nach einander die Zahlen 1, 2, 3, so erhält man, mit Berücksichtigung von (XII.),

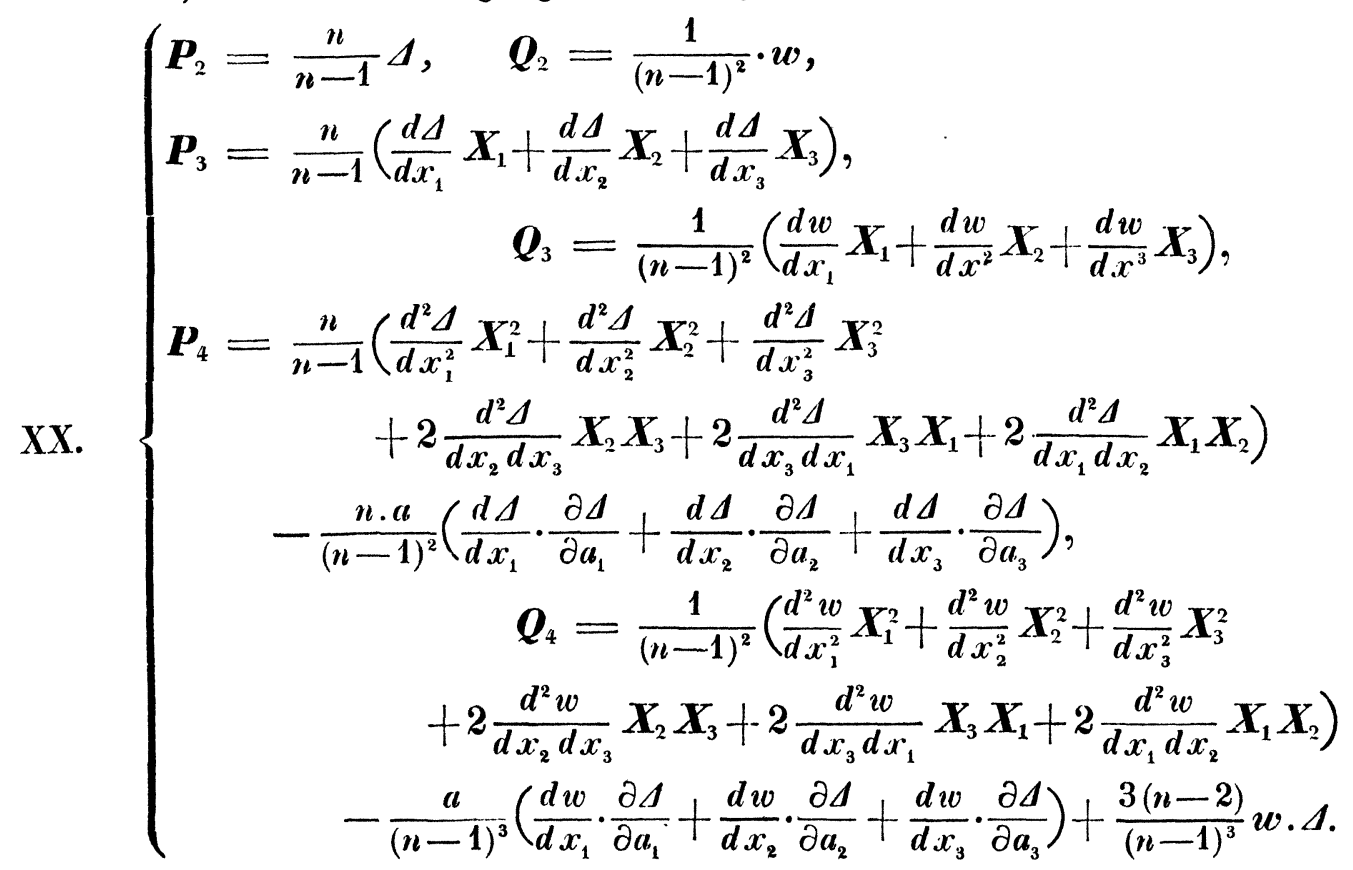

Die angegebenen Werthe von $\boldsymbol{P}_{2}$ und $\boldsymbol{Q}_{2}$ sind wichtig bei der Bestimmung der Wendepuncte einer Curve nter Ordnung. Denn läfst man $x_{1}, x_{2}, x_{3}$ die Coordinaten eines Wendepuncts der Curve $v=0$ bedeuten, so müssen die drei ersten Glieder der Reihe [v] für alle Werthe von $a_{1}, a_{2}, a_{3}$ verschwinden. Da aber das zweite Glied von selbst verschwindet, so bleiben zur Bestimmung der Wendepuncte die beiden Gleichungen:

$$
(v)=0, \quad\left(\partial^{2} v\right)=0
$$


übrig, welche für alle Werthe von $a_{1}, a_{2}, a_{3}$ erfüllt werden sollen. Da nun $\left(\partial^{2} v\right)=\boldsymbol{P}_{2} v-\boldsymbol{Q}_{2} a^{2}$ ist, so erhält man zur Bestimmung der Wendepuncte :

$$
v=0, \quad(n-1)^{2} Q_{2}=w=0 ;
$$

welche Gleichungen ich in Bd. 28. dieses Journals S. 104 Lehrsatz 9. aufgestellt habe.

Auf der Zurückführung des Ausdrucks $\left(\partial^{3} v\right)$, in dem Falle $n=3$, auf die Form $\left(\partial^{3} \boldsymbol{v}\right)=\boldsymbol{P}_{3} \boldsymbol{v}-\boldsymbol{Q}_{3} a^{2}$, wo $\boldsymbol{P}_{3}$ und $\boldsymbol{Q}_{3}$ die angegebenen Werthe haben, beruht der Beweis des Lehrsatzes (17.). Denn setzt man in den Ausdruck $\boldsymbol{Q}_{3}$ die Werthe von $\boldsymbol{X}_{1}, \boldsymbol{X}_{2}, \boldsymbol{X}_{3}$ aus (VI.), so geht derselbe in den Theil links der Gleichung (III.) über.

Auf eben dieser Zurückführung, in dem Falle $n=4$, beruht der Beweis des folgenden Lehrsatzes:

Wenn eine Curve Ater Ordnung $v=0$ und eine gerade Linie $a=0$ gegeben sind, so giebt es 32 Tangenten der Curve, welche von der Curve in swei Punclen geschnilten werden, die harmonisch sind su dem Berührungspuncte und den Schnittpuncte der Tangente mit der gegebenen geraden Linie. Die Berührungspuncte der 32 Tangenten wierden durch die beiden Gleichungen

$$
\begin{gathered}
v=0 \text { und } \frac{\partial a_{1}}{\partial x_{1}}\left(\frac{\partial v}{\partial x_{2}} \cdot \frac{\partial w}{\partial x_{3}}-\frac{\partial v}{\partial x_{3}} \cdot \frac{\partial w}{\partial x_{2}}\right)+\frac{\partial a_{2}}{\partial x_{2}}\left(\frac{\partial v}{\partial x_{3}} \cdot \frac{\partial w}{\partial x_{1}}-\frac{\partial v}{\partial x_{1}} \cdot \frac{\partial w}{\partial x_{3}}\right) \\
+\frac{\partial a_{3}}{\partial \lambda_{3}}\left(\frac{\partial v}{\partial x_{1}} \cdot \frac{\partial w}{\partial x_{2}}-\frac{\partial v}{\partial x_{2}} \cdot \frac{\partial w}{\partial x_{1}}\right)=0
\end{gathered}
$$

beslimmt; wo $w$ die Determinante der Function $v$ bedeutet.

Die Reihe $[v]$ ist eine homogene Function der Gröfsen $x_{1}+\lambda X_{1}$, $x_{2}+\lambda \boldsymbol{X}_{2}$ und $x_{3}+\lambda \boldsymbol{X}_{3}$. Betrachtet man diese drei Gröfsen als die Coordinaten eines Puncts, so wird dieser Punct auf einer und derselben geraden Linie liegen, wie man auch die Gröfse $\lambda$ sich verändern lasse. Diese gerade Linie wird zur Tangente der Curve $v=0$ in dem Puncte $x_{1} x_{2} x_{3}$, wenn dieser Punct in die Curve selbst hineinrückt, also das erste Glied der Reihe verschwindet. Denn das zweite Glied verschwindet, wie oben bemerkt, von selbst, da $\boldsymbol{X}_{1}, \boldsymbol{X}_{2}, \boldsymbol{X}_{3}$ die Bedeutung (VI.) haben. Unter dieser Voraussetzung wird nun die Tangente eine Doppeltangente, wenn die Gleichung $[v]=0$ aufser ihren beiden gleichen Wurzeln $\lambda=0$ noch zwei andere gleiche Wurzeln hat; und die Bedingungsgleichung, welche zu erfüllen ist, damit dieses zutreffe, wird die Curve darstellen, welche die gegebene Curve $v=0$ in solchen Fällen schneidet, in welchen die Tangenten zugleich Doppeltangenten der 
gegebenen Curve sind. Um diese Bedingungsgleichung aufzustellen, lasse ich aus der Gleichung $[v]=0$ die beiden ersten verschwindenden Glieder weg und dividire mit $\lambda^{2}$. Dies giebt:

$$
\frac{\left(\partial^{2} v\right)}{1.2}+\lambda \cdot \frac{\left(\partial^{3} v\right)}{1.2 .3}+\lambda^{2} \cdot \frac{\left(\partial^{4} v\right)}{1.2 .3 .4}+\ldots \lambda^{n-2} \cdot \frac{\left(\partial^{n} v\right)}{1.2 \ldots n}=0 .
$$

Da aber $\left(\partial^{\mu} v\right)=\boldsymbol{P}_{\mu} \cdot v-\boldsymbol{Q}_{\mu} \cdot a^{2}$ ist, so nimmt diese Gleichung, wenn man der Kürze wegen

XXI. $\quad[[v]]=\frac{Q_{2}}{1.2}+\lambda \cdot \frac{Q_{3}}{1.2 .3}+\lambda^{2} \cdot \frac{Q_{4}}{1.2 .3 .4}+\ldots . \lambda^{n-2} \cdot \frac{Q_{n}}{1.2 \ldots . n} \ldots$ setzt, wegen $v=0$, die einfachere Gestalt:

$$
\text { XXII. }[[v]]=0
$$

an. Damit die Gleichung zwei gleiche Wurzeln habe, mufs man durch denselben Werth von $\lambda$ folgenden beiden Gleichungen genügen können:

XXIII. $\left\{\begin{array}{l}\frac{(n-2) Q_{2}}{1.2}+\frac{(n-3) Q_{3}}{1.2 .3} \cdot \lambda+\ldots \frac{2 . Q_{n-2}}{1.2 \ldots(n-2)} \cdot \lambda^{n-4}+\frac{Q_{n-1}}{1.2 \ldots(n-1)} \cdot \lambda^{n-3}=0, \\ \frac{Q_{3}}{1.2 .3}+\frac{2 . Q_{4}}{1.2 .3 .4} \cdot \lambda+\ldots \frac{(n-3) Q_{n-1}}{1.2 \ldots .(n-1)} \cdot \lambda^{n-4}+\frac{(n-2) Q_{n}}{1.2 \ldots . n} \cdot \lambda^{n-3}=0 .\end{array}\right.$

Eliminirt man $\lambda$ aus diesen beiden Gleichungen, so erhält man die gesuchte Bedingungsgleichung

$$
\text { XXIV. } \quad R=0 \text {, }
$$

welche, da sie die unbestimmten Gröfsen $a_{1}, a_{2}, a_{3}$ enthält, ein ganzes System von Curven von der $(n+4)(n-2)(n-3)$ ten Ordnung darstell, welche sämmtlich durch die Berührungspuncte der Doppeltangenten hindurchgehen. Denn man sieht, dafs die Function $\boldsymbol{R}$ homogen ist und:

Vom Grade $(n-2)(n-3)$ in Beziehung auf die Gröfsen $a_{1}, a_{2}, a_{3}$,

Vom Grade $(n+4)(n-3)$ in Beziehung auf die Coëfficienten $v$ und

Vom Grade $(n+4)(n-2)(n-3)$ in Beziehung auf die Variabeln $x_{1}, x_{2}, x_{3}$.

Wenn man in (XXIII.) $\left(\partial^{2} v\right),\left(\partial^{3} v\right), \ldots$ statt $\boldsymbol{Q}_{2}, \boldsymbol{Q}_{3}, \ldots$ setzt, so ist das Resultat der Elimination von $\lambda$ aus den beiden Gleichungen eine homogene Gleichung von den Graden $(n+2)(n-3),(n+4)(n-3),\left(n^{2}+2 n-4\right)(n-3)$ in Beziehung auf die Gröfsen $a_{1}, a_{2}, a_{3}$, die Coëfficienten in $v$ und die Variabeln $x_{1}, x_{2}, x_{3}$; und diese Gleichung stellt, wie die vorhergehende, eine Curve dar, welche durch die Berührungspuncte der Doppeltangenten hindurchgeht. Es ist dieses dieselbe Gleichung, welche Herr Cayley (Bd. 34. dieses Journals S. 37) durch

$$
[\boldsymbol{Y}]=\mathbf{0}
$$


bezeichnet hat. Der Grad derselben läfst sich mit Hülfe der gegebenen Gleichung $v=0$ der Curve um $4(n-3)$ Einheiten verringern. Denn da, wie aus dem Vorhergehenden ersichtlich,

$$
[\boldsymbol{Y}]=\boldsymbol{T} \cdot \boldsymbol{v}-\boldsymbol{R} \cdot \boldsymbol{a}^{4(n-3)}
$$

ist, wo $\boldsymbol{T}$ eine homogene Function von den Graden

$$
(n+2)(n-3), \quad(n+4)(n-3)-1, \quad\left(n^{2}+2 n-4\right)(n-3)-n
$$

in Beziehung auf die Gröfsen $a_{1}, a_{2}, a_{3}$, die Coëfficienten in $v$ und die Variabeln $x_{1}, x_{2}, x_{3}$ bezeichnet, so reducirt sich die Gleichung, mit Zuziehung der Gleichung $v=0$, auf die Gleichung (XXIV.).

Es bleibt noch übrig, auf ähnliche Weise den Grad der letztern Gleichung $\boldsymbol{R}=0$ um $(n-2)(n-3)$ Einheiten zu verringern, um das Problem der Doppeltangenten vollständig zu lösen; was mir aber nicht hat gelingen wollen; selbst nicht in dem einfachsten Falle, wenn die gegebene Curve vom 4ten Grade ist. In diesem Falle nimmt, wenn man in (XX.) $n=4$ setzt, die Gleichung (XXIV.) die einfache Gestalt

$$
\text { XXV. } \quad 3 \cdot Q_{2} \cdot Q_{4}-Q_{3} \cdot Q_{3}=0
$$

an, deren Grad mit Hülfe der gegebenen Gleichung $v=0$ noch um zwei Einheiten zu verringern bleibt.

Nach diesen Bemerkungen über die Doppeltangenten der Curven, zu welchen der Lehrsatz (17.) Veranlassung gab, kehre ich zu dem Systeme von Gleichungen (1.) zurück, von welchen die vorliegende Untersuchung der Curven 3ter Ordnung ausging.

4.

Aus dem Systeme von Gleichungen (1.) lassen sich andere Gleichungen von analytischem Interesse ableiten. Setzt man zu diesem Ende

$$
\begin{aligned}
& \boldsymbol{u}_{2,2} \boldsymbol{u}_{3,3}-\boldsymbol{u}_{2,3}^{2}=\boldsymbol{a}_{1,1}, \quad \boldsymbol{u}_{1,2} \boldsymbol{u}_{1,3}-\boldsymbol{u}_{1,1} \boldsymbol{u}_{2,3}=a_{2,3}=a_{3,2}, \\
& \boldsymbol{u}_{3,3} \boldsymbol{u}_{1,1}-u_{3,1}^{2}=a_{2,2}, \quad \boldsymbol{u}_{2,3} \boldsymbol{u}_{2, \mathbf{1}}-\boldsymbol{u}_{2,2} \boldsymbol{u}_{3, \mathbf{1}}=\boldsymbol{a}_{3,1}=\boldsymbol{a}_{1,3}, \\
& u_{1,1} u_{2,2}-u_{1,2}^{2}=a_{3,3}, \quad u_{3,1} u_{3,2}-u_{3,3} u_{1,2}=a_{1,2}=a_{2,1} \text {, }
\end{aligned}
$$

und bestimmt das Verhältnifs der Coordinaten $\boldsymbol{X}_{1}, \boldsymbol{X}_{2}, \boldsymbol{X}_{\mathbf{3}}$ des Puncts $\boldsymbol{P}$ aus je zwei Gleichungen (1.), so erhält man

$$
X_{1}: X_{2}: X_{3}=a_{1,1}: a_{1,2}: a_{1,3}=a_{2,1}: a_{2,2}: a_{2,3}=a_{3,1}: a_{3,2}: a_{3,3}
$$

woraus sich, wenn man durch $\varrho$ einen unbestimmten Factor bezeichnet, die Gleichungen

$$
\text { 2. } \quad\left\{\begin{array}{lll}
X_{1} X_{1}=\rho a_{1,1}, & X_{2} X_{2}=\rho a_{2,2}, & X_{3} X_{3}=\rho a_{3,3}, \\
X_{2} X_{3}=\rho a_{2,3}, & X_{3} X_{1}=\rho a_{3,1}, & X_{1} X_{2}=\rho a_{1,2}
\end{array}\right.
$$

ableiten lassen. Da sich in den Gleichungen (1.) die Coordinaten $x_{1}, x_{2}, x_{3}$ des 
Punctes $\boldsymbol{p}$ mit den Coordinaten $\boldsymbol{X}_{1}, \boldsymbol{X}_{2}, \boldsymbol{X}_{3}$ des Punctes $\boldsymbol{P}$ vertauschen lassen, so ist dieses auch in den von ihnen abgeleiteten Gleichungen (2.) gestattet. Bezeichnet man nun durch $\boldsymbol{A}$ die Ausdrücke, in welche die homogenen Functionen 2 ten Grades $a$ in dem Theile rechts der Gleichungen (2.) übergehen, wenn $\operatorname{man} \boldsymbol{X}_{1}, \boldsymbol{X}_{2}, \boldsymbol{X}_{3}$ statt $x_{1}, x_{2}, x_{3}$ setzt, und läfst $\boldsymbol{P}$ einen unbestimmten Factor bedeuten, so folgt aus den Gleichungen (2.) durch jene Vertauschung:

$$
\text { 3. }\left\{\begin{array}{lll}
x_{1} x_{1}=\boldsymbol{P} A_{1,1}, & x_{2} x_{2}=\boldsymbol{P} A_{2,2}, & x_{3} x_{3}=\boldsymbol{P} A_{3,3}, \\
x_{2} x_{3}=\boldsymbol{P} A_{2,3}, & x_{3} x_{1}=\boldsymbol{P} A_{3,1}, & x_{1} x_{2}=\boldsymbol{P} A_{1,2} .
\end{array}\right.
$$

Dieses System von Gleichungen steht mit dem Systeme von Gleichungen (2.) in einer merkwürdigen Verbindung. Betrachtet man nemlich die in den Theilen rechts der Gleichungen (2.) lineär vorkommenden 6 Producte $x_{1} x_{1}, x_{2} x_{2}, x_{3} x_{3}$, $x_{2} x_{3}, x_{3} x_{1}, x_{1} x_{2}$ àls Unbekannte und löset das System von Gleichungen nach diesen Unbekannten auf, so erhält man das System von Gleichungen (3.), mit denselben Coëfficienten der Producte $\boldsymbol{X}_{1} \boldsymbol{X}_{1}, \boldsymbol{X}_{2} \boldsymbol{X}_{2}, \boldsymbol{X}_{3} \boldsymbol{X}_{3}, \boldsymbol{X}_{2} \boldsymbol{X}_{3}, \boldsymbol{X}_{3} \boldsymbol{X}_{1}, \boldsymbol{X}_{1} \boldsymbol{X}_{2}$, mit welchen in (2.) die Producte $x_{1} x_{1}, x_{2} x_{2}, \ldots \ldots x_{1} x_{2}$ multiplicirt sind, abgesehen von einem gemeinschaftlichen Factor.

Jeden 6 Puncten $P$ der Curve 3ter Ordnung $\varphi=0$, durch welche sich ein Kegelschnitt legen lä/st, entsprechen 6 Puncte $p$, welche auf der Curve und zugleich auf einem andern Kegelschnitt liegen. Denn wenn die Gleichung des ersten Kegelschnitts

4. $\alpha_{1,1} \boldsymbol{X}_{1} \boldsymbol{X}_{1}+\alpha_{2,2} \boldsymbol{X}_{2} \boldsymbol{X}_{2}+\alpha_{3,3} \boldsymbol{X}_{3} \boldsymbol{X}_{3}+2 \alpha_{2,3} \boldsymbol{X}_{2} \boldsymbol{X}_{3}+2 \alpha_{3,1} \boldsymbol{X}_{3} \boldsymbol{X}_{1}+2 \alpha_{1,2} \boldsymbol{X}_{1} \boldsymbol{X}_{2}=0$ ist, so erhellet aus den Gleichungen (2.), dafs die Gleichung des zweiten Kegelschnitts

5. $\quad \alpha_{1,1} a_{1,1}+\alpha_{2,2} a_{2,2}+\alpha_{3,3} a_{3,3}+2 \alpha_{2,3} a_{2,3}+2 \alpha_{3,1} a_{3,1}+2 \alpha_{1,2} a_{1,2}=0$ sein wird. Geht der erste Kegelschnitt in ein Linienpaar $\boldsymbol{A}, \boldsymbol{B}$ über, dessen Gleichung

$$
\text { 6. }\left(\alpha_{1} \boldsymbol{X}_{1}+\alpha_{2} \boldsymbol{X}_{2}+\alpha_{3} \boldsymbol{X}_{3}\right)\left(\beta_{1} \boldsymbol{X}_{1}+\beta_{2} \boldsymbol{X}_{2}+\beta_{3} \boldsymbol{X}_{3}\right)=0
$$

ist, so ist die Gleichung des zweiten Kegelschnitts

$$
\text { 7. } \begin{aligned}
\alpha_{1} \beta_{1} a_{1,1}+\alpha_{2} \beta_{2} a_{2,2}+\alpha_{3} \beta_{3} a_{3,3}+\left(\alpha_{2} \beta_{3}+\alpha_{3} \beta_{2}\right) a_{2,3}+\left(\alpha_{3} \beta_{1}+\alpha_{1} \beta_{3}\right) a_{3,1} \\
+\left(\alpha_{1} \beta_{2}+\alpha_{2} \beta_{1}\right) a_{1,2}=0 .
\end{aligned}
$$

Läfst man die zweite Linie $\boldsymbol{B}$ des Linienpaares sich der ersten $\boldsymbol{A}$ so lange nähern, bis sie mit ihr zusammenfällt, so geht die Gleichung (6.) in

$$
\text { 8. }\left(\alpha_{1} \boldsymbol{X}_{1}+\alpha_{2} \boldsymbol{X}_{2}+\alpha_{3} \boldsymbol{X}_{3}\right)^{2}=0
$$

über, während die Gleichung des zweiten Kegelschnitts die Form

9. $\quad \alpha_{1} \alpha_{1} a_{1,1}+\alpha_{2} \alpha_{2} a_{2,2}+\alpha_{3} \alpha_{3} a_{3,3}+2 \alpha_{2} \alpha_{3} a_{2,3}+2 \alpha_{3} \alpha_{1} a_{3,1}+2 \alpha_{1} \alpha_{2} a_{1,2}=0$ annimmt. Dieses ist aber die Gleichung eines Kegelschnitts, welcher die Curve 
dritter Ordnung in drei verschiedenen Puncten berührt. Denn da die Schnittpuncte $\boldsymbol{P}$ der Curve dritter Ordnung und des Linienpaares (8.) paarweise zusammenfallen, so werden auch die ihnen entsprechenden Schnittpuncte $\boldsymbol{p}$ des Kegelschnitts (9.) und der Curve paarweise zusammenfallen, oder, was dasselbe ist, der Kegelschnitt wird die Curve in drei verschiedenen Puncten berühren.

Betrachtet man in der Gleichung (9.) die Constanten $\alpha_{1}, \alpha_{2}, \alpha_{3}$ als variabel, so drückt die genannte Gleichung ein ganzes System von Kegelschnitten aus, deren jeden die Curve in drei verschiedenen Puncten berührt. Es entsprechen also jeden drei Puncten $P$ der Curve dritter Ordnung, welche in einer geraden Linie liegen, drei Puncte $p$, in welchen ein Kegelschnitt die Curve berührt. Dieses sind jedoch nicht alle Kegelschnitte von der genannten Eigenschaft. Denn da in die Gleichung (9.) die Coëfficienten aus einer der drei Functionen $f$ eingehen, so wird jeder dieser Functionen ein System solcher Kegelschnitte entsprechen. Die Gleichung (9.) umfafst also drei verschiedene, den drei Functionen $f$ entsprechende Gleichungen; und diese letztern sind die allgemeinen analytischen Ausdrücke für die drei Systeme von Kegelschnitten, welche die Curve in drei verschiedenen Puncten berühren. Es entsprechen also jeden drei Puncten $\boldsymbol{P}$ der Curve dritter Ordnung, welche in einer geraden Linie liegen, 3 Systeme von 3 Puncten $p$, welche die Berührungspuncte dreier Kegelschnitte und der Curve sind. Da die drei Puncte $\boldsymbol{P}$ und die 9 Puncte $p$ als die Berührungspuncte der 12 Tangenten angesehen werden können, welche von 3 leicht zu bestimmenden, auf der Curve und einer geraden Linie gelegenen Puncten an die Curve gezogen werden, so liegen von diesen 12 Puncten $16 \mathrm{mal}$ drei Puncte in einer geraden Linie, und von den 9 Puncten $p$, 6mal drei Puncte in einer geraden Linie.

Dafs die erwähnten drei Systeme vom Kegelschnitte (9.) alle Kegelschnitte umfassen, welche die gegebene Curve in drei verschiedenen Puncten berühren, läfst sich durch folgende Betrachtung nachweisen. Es seien $p_{1}, p_{2}$, $p_{3}$ die Berührungspuncte eines Kegelschnitts und der Curve. Die drei Tangenten $\pi_{1}, \pi_{2}, \pi_{3}$ in diesen Puncten lassen sich als eine Curve 3ter Ordnung betrachten. Da dieselben aber die gegebene Curve in 9 Puncten schneiden, von welchen 6 in einem Kegelschnitte liegen, so müssen die drei andern in einer geraden Linie liegen. Demnach erhält man, wenn die beiden Berührungspuncte $p_{1}$ und $p_{2}$ auf der Curve beliebig gegeben sind, den dritten $p_{3}$, wenn man die Tangenten $\pi_{1}, \pi_{2}$ construirt (welche die Curve in den Puncten 
$q_{1}$ und $q_{2}$ schneiden mögen), hierauf die gerade Linie $q_{1} q_{2}$ zieht, welche der Curve in dem Puncte $q_{3}$ begegnet und von diesem Puncte $q_{3}$ an die Curve eine Tangente zieht. Der Berührungspunct $p_{3}$ wird dann der gesuchte Punct sein. Nun lassen sich aber von dem Puncte $q_{3}$ der Curve 4 Tangenten an die Curve ziehen, von denen eine die Curve in dem Schnittpuncte der geraden Linie $p_{1} p_{2}$ und der Curve berührt. Dieser Punct mufs aber verworfen werden, weil unter dem Kegelschnitte, welcher die Curve in den Puncten berührt, die in einer geraden Linie liegen, nur ein Linienpaar verstanden werden kann, welches mit der geraden Linie zusammenfällt. Man hat also in der That nur 3 Kegelschnitte, welche die gegebene Curve in 3 verschiedenen Puncten berühren, von denen 2 auf der Curve beliebig gegeben sind. Diese 3 Kegelschnitte finden sich aber auch unter den Kegelschnitten (9.). Denn wenn $\boldsymbol{P}_{1}, \boldsymbol{P}_{2}$ die den Puncten $p_{1}, p_{2}$ entsprechenden Puncte in einem der 3 Systeme sind und man bestimmt den dem Schnittpuncte $\boldsymbol{P}_{3}$ der geraden Linie $\boldsymbol{P}_{1} \boldsymbol{P}_{2}$ und der Curve entsprechenden Punct $\boldsymbol{p}_{3}$ in demselben Systeme, so hat man den dritten Berührungspunct des Kegelschnitts, welcher die Curve in den Puncten $p_{1}, p_{2}$ berührt. Diese Construction, für die drei Systeme ausgeführt, giebt ebenfalls 3 Kegelschnitte von der genannten Eigenschaft und welche nur jene 3 vorhin erwähnten sein können. Dieses läfst sich kurz wie folgt zusammenfassen.

17. Alle Kegelschnitte, welche eine gegebene Curve dritter Ordnung in drei verschiedenen Puncten berühren, ordnen sich dreien Systemen unter. Ein gegebener Kegelschnitt dieser Art gehört dem einen oder dem andern Systeme an, je nachdem ein Berührungspunct und der Schnitlpunct der geraden Linie (welche die beiden andern verbindel) und der Curve, ein Polenpaar aus dem einen oder dem andern Systeme bilden.

Auf dieselbe Weise, wie den 3 Schnittpuncten $\boldsymbol{P}^{\prime}, \boldsymbol{P}^{\prime \prime}, \boldsymbol{P}^{\prime \prime \prime}$ der geraden Linie $\boldsymbol{A}$ und der Curve dritter Ordnung 3 Puncte $\boldsymbol{p}^{\prime}, \boldsymbol{p}^{\prime \prime}, \boldsymbol{p}^{\prime \prime \prime}$ entsprechen, in welchen der Kegelschnitt (9.) die Curve berührt, entsprechen auch den 3 Schnittpuncten $\boldsymbol{P}^{(4)}, \boldsymbol{P}^{(5)}, \boldsymbol{P}^{(6)}$ der geraden Linie $\boldsymbol{B}$ und der Curve 3 Puncte $\boldsymbol{p}^{(4)}, \boldsymbol{p}^{(5)}, \boldsymbol{p}^{(6)}$, in welchen der Kegelschnitt

10. $\beta_{1} \beta_{1} a_{1,1}+\beta_{2} \beta_{2} a_{2,2}+\beta_{3} \beta_{3} a_{3,3}+2 \beta_{2} \beta_{3} a_{2,3}+2 \beta_{3} \beta_{1} a_{3,1}+2 \beta_{1} \beta_{2} a_{1,2}=0$ die Curve berūhrt. Da aber das Linienpaar $\boldsymbol{A}, \boldsymbol{B}$, welches durch die Gleichung (6.) dargestellt ist, die Curve in den 6 Puncten $\boldsymbol{P}$ schneidet, so geht der Kegelschnitt (7.) durch die diesen entsprechenden 6 Puncte $p$. Dieses läfst sich umgekehrt auch so ausdrücken: 
18. Wenn man durch die drei Tangirungspuncte eines Kegelschnitts und einer Curve dritter Ordnung einen beliebigen Kegelschnitt legt, so schneidet derselbe die Curve in drei neuen Puncten, in welchen ein anderer Kegelschnitt die Curve berührt.

Dafs dieser berührende Kegelschnitt zu demselben System wie der erste berührende Kegelschnitt gehört, ist einleuchtend. Es ist auch noch zu bemerken, dafs, wenn man den beliebigen Kegelschnitt, welcher durch die drei Berührungspuncte eines Kegelschnitts und der Curve gelegt ist, um die drei Berührungspuncte auf alle mögliche Art variiren läfst, unter den 3 Schnittpuncten desselben mit der Curve auch die 3 Tangirungspuncte eines beliebigen berührenden Kegelschnitts aus demselben Systeme sein werden.

5.

Um auch die speciellen Fälle der Curven dritter Ordnung in unsere Betrachtungen zu ziehen, bemerken wir, dafs, während sich die Gleichung der Curve 3ter Ordnung $\varphi=0$, wie in (Bd. 28. dieses Journals S. 90) gezeigt, im Allgemeinen auf die Form

$$
\text { 11. } \varphi=y_{1}^{3}+y_{2}^{3}+y_{3}^{3}-2 \pi y_{1} y_{2} y_{3}=0
$$

zurückführen läfst, wo $y_{1}=0, y_{2}=0, y_{3}=0$ die Gleichungen dreier geraden Linien bedeuten, welche durch die 9 Wendepuncte der Curve hindurchgehen, in dem besonderen Falle, wenn die Curve einen Doppelpunct hat, aus jener Gleichung eins der drei ersten Glieder wegfält, so dafs die Gleichung der Curve die Gestalt

$$
\text { 12. } \varphi=y_{1}^{3}+y_{2}^{3}-2 \pi y_{1} y_{2} y_{3}=0
$$

annimmt. Denn es ist leicht zu sehen, dafs die Gleichung der Curve diese Form annehmen mufs, wenn $y_{1}=0, y_{2}=0$ die Gleichungen der beiden Tangenten in dem Doppelpuncte der Curve sind. Stellt man, um die Wendepuncte der Curve zu finden, welche einen Doppelpunct hat, von der Function $\varphi$ die Determinante

$$
\psi=-24 \pi^{2}\left\{y_{1}^{3}+y_{2}^{3}+\frac{2}{3} \pi y_{1} y_{2} y_{3}\right\}
$$

auf, so erhălt man für diese Puncte:

$$
\varphi=0, \quad \psi=0
$$

woraus ersichtlich ist, dafs 6 von den 9 Wendepuncten in den Doppelpunct fallen, während die drei andern in der geraden Linie $y_{3}=0$ liegen.

Von den 4 Tangenten, welche von einem beliebigen Puncte der Curve an die Curve gezogen werden können, fallen zwei mit derjenigen geraden 
Linie zusammen, welche den beliebigen Punct der Curve mit dem Doppelpuncte verbindet. Da diese gerade Linie aber nicht als eine wahre Tangente zu betrachten ist, so bleiben in der That nur 2 Tangenten übrig, und die Tangirungspuncte $\boldsymbol{p}$ und $\boldsymbol{P}$ sind zwei entsprechende Pole in dem einzigen hier Statt findenden Systeme. Die Function $f$ für diesen Fall ist, abgesehen von einem constanten Factor, auf welchen es nicht ankommt:

$$
f=y_{1}^{3}+y_{2}^{3}+6 \pi y_{1} y_{2} y_{3} \text {. }
$$

Wenn man durch $\boldsymbol{Y}_{1}, \boldsymbol{Y}_{2}, \boldsymbol{Y}_{3}$ dieselben lineären Functionen der Variabeln $\boldsymbol{X}_{1}, \boldsymbol{X}_{2}, \boldsymbol{X}_{3}$ bezeichnet, welche $y_{1}, y_{2}, y_{3}$ von $x_{1}, x_{2}, x_{3}$ sind, so ergiebt sich folgendes System von Gleichungen zwischen den Coordinaten $x_{1}, x_{2}, x_{3}$ und $\boldsymbol{X}_{1}, \boldsymbol{X}_{2}, \boldsymbol{X}_{3}$ der entsprechenden Pole $\boldsymbol{p}$ und $\boldsymbol{P}$, welches dann in dem vorliegenden Falle das System von Gleichungen (1.) vertritt:

$$
\begin{aligned}
& \boldsymbol{Y}_{1} y_{1}+\boldsymbol{Y}_{2} \pi y_{3}+\boldsymbol{Y}_{3} \pi y_{2}=0 \\
& \boldsymbol{Y}_{1} \pi y_{3}+\boldsymbol{Y}_{2} y_{2}+\boldsymbol{Y}_{3} \pi y_{1}=0, \\
& \boldsymbol{Y}_{1} y_{2}+\boldsymbol{Y}_{2} y_{1}+0=0 .
\end{aligned}
$$

Aus der letzten dieser Gleichungen ist zu sehen, dafs die Verbindungslinien der beiden entsprechenden Pole $\boldsymbol{p}$ und $\boldsymbol{P}$ mit dem Doppelpuncte harmonisch sind zu den beiden Tangenten der Curve in dem Doppelpuncte. Man erhält also den einern gegebenen Puncte $\boldsymbol{P}$ der. Curve entsprechenden Pol $p$, wenn man $\boldsymbol{P}$ mit dem Doppelpuncte der Curve durch eine gerade Linie verbindet und $\mathbf{s u}$ dieser Verbindungslinie und dem Tangentenpaare in dem Doppelpuncte die vierte harmonische Linie construirt. Diese Linie schneidet die Curve in dem Puncte $p$.

Wenn drei Puncte $\boldsymbol{P}$ der Curve auf einer geraden Linie $\boldsymbol{A}$ liegen, deren Gleichung

$$
\text { 13. } \alpha_{1} \boldsymbol{Y}_{1}+\alpha_{2} \boldsymbol{Y}_{2}+\alpha_{3} \boldsymbol{Y}_{3}=0
$$

ist, so berührt der Kegelschnitt

14. $\alpha_{1} \alpha_{1} y_{1}^{2}+\alpha_{2} \alpha_{2} y_{2}^{2}+\alpha_{3} \alpha_{3} y_{3}^{2}-2 \alpha_{2} \alpha_{3} y_{2} y_{3}-2 \alpha_{3} \alpha_{1} y_{3} y_{1}-2 \alpha_{1} \alpha_{2} y_{1} y_{2}$

$$
-\frac{\alpha_{3}}{\pi^{2}}\left\{\alpha_{3} y_{1} y_{2}-2 \alpha_{2} \pi y_{1}^{2}-\alpha_{1} \pi y_{2}^{2}\right\}=0
$$

die Curve in den drei den Puncten $\boldsymbol{P}$ entsprechenden Polen $\boldsymbol{p}$, welche dem Vorhergehenden zufolge von dem Doppelpuncte aus leicht sich construiren lassen. Und diese Gleichung, welche analog der Gleichung (10.) gebildet ist, stellt, wenn man $\alpha_{1}, \alpha_{2}, \alpha_{3}$ beliebig variiren läfst, das ganze System der Kegelschnitte dar, welche die Curve dritter Ordnung, mit einem Doppelpuncte, in drei verschiedenen Puncten berühren. 
Wenn, zweitens, die betrachtete Curve zwei Doppelpuncte hat, so fallen in der Gleichung (11.) zwei von den drei ersten Gliedern weg und die Curve 15. $\varphi=y_{1}^{3}-2 \pi y_{1} y_{2} y_{3}=0$

zerfällt in einen Kegelschnitt $y_{1}^{2}-2 \pi y_{2} y_{3}=0$ und in eine gerade Linie $y_{1}=0$. Letztere schneidet den Kegelschnitt in den beiden Doppelpuncten der Curve. Die Tangenten des Kegelschnitts in diesen beiden Puncten werden durch die Gleichungen $y_{2}=0, y_{3}=0$ ausgedrückt. Jedem Puncte $\boldsymbol{P}$ der Curve entspricht nun ein $\mathrm{Pol} p$, dessen Coordinaten folgenden Relationen unterworfen sind:

$$
\begin{aligned}
& \boldsymbol{Y} y_{1}+\boldsymbol{Y}_{2} \pi y_{3}+\boldsymbol{Y}_{3} \pi y_{2}=0, \\
& \boldsymbol{Y}_{1} y_{3}+0, \boldsymbol{Y}_{3} y_{1}=0, \\
& \boldsymbol{Y}_{1} y_{2}+\boldsymbol{Y}_{2} y_{1}+0=0 .
\end{aligned}
$$

Aus den beiden letzten Gleichungen sieht man, da/s das Linienpaar, welches zwei entsprechende Pole $\boldsymbol{P}$ und $p$ mit einem der Doppelpuncte verbindet, zu der Tangente in dem Doppelpuncte und der Verbindungslinie der beiden Doppelpuncte harmonisch ist. Hiernach läfst sich, wenn ein Punct $\boldsymbol{P}$ des Kegelschnitts gegeben ist, der entsprechende Pol $\boldsymbol{p}$ der Curve dritter Ordnung construiren; welcher ebenfalls auf dem Kegelschnitte liegen wird. Aus den beiden genannten Gleichungen entnimmt man ferner, da/s wenn der Punct $\boldsymbol{P}$ auf der Verbindungslinie der beiden Doppelpuncte liegt, der entsprechende Pol $p$ ebenfalls auf dieser geraden Linie liegen wird; und aus der ersten Gleichung, dafs in diesem Falle das Polenpaar $p, p$ harmonisch ist zu den beiden Doppelpuncten. Durchschneidet man die Curve mit einer geraden Linie A:

$$
\text { 16. } \alpha_{1} Y_{1}+\alpha_{2} Y_{2}+\alpha_{3} Y_{3}=0 \text {, }
$$

in drei Puncten $\boldsymbol{P}$, so lassen sich nach den obigen Bemerkungen die entsprechenden Pole $\boldsymbol{p}$ leicht construiren. In diesen Polen $\boldsymbol{p}$ berührt nun der Kegelschnitt, dessen Gleichung

17. $\alpha_{1} \alpha_{1} y_{1}^{2}+\alpha_{2} \alpha_{2} y_{2}^{2}+\alpha_{3} \alpha_{3} y_{3}^{2}-2 \alpha_{2} \alpha_{3} y_{2} y_{3}-2 \alpha_{3} \alpha_{1} y_{3} y_{1}-2 \alpha_{1} \alpha_{2} y_{1} y_{2}$

$$
+\frac{2 \alpha_{2} \alpha_{3} y_{1}^{2}}{\pi}=0
$$

ist, den Kegelschnitt zweimal und die gerade Linie, aus welchen die Curve besteht. Es ist hier noch zu bemerken, dafs die Gleichung (17.), wenn man darin $\alpha_{1}, \alpha_{2}, \alpha_{3}$ variiren läfst, alle Kegelschnitte umfafst, welche den Kegelschnitt und die gerade Linie, ans welchen die Curve 3ter Ordnung besteht, erstere in zwei verschiedenen Puncten, letztere in einem Puncte berühren.

Crelle's Journal f. d.M. Bd. XXXVI. Heft 2. 
Hieran knüpft sich nun die Lösung der nachstehenden Aufgabe. „Wenn „ein Kegelschnitt und eine gerade Linie gegeben sind: die Berührungspuncte „desjenigen Kegelschnitts zu construiren, welcher den gegebenen Kegelschnitt ,in zwei Puncten und die gegebene gerade linie in einem Puncte berührt; sei „es, dafs die beiden Berührungspuncte auf dem gegebenen Kegelschnitt, oder „ein Berührungspunct auf dem Kegelschnitt, der andere auf der geraden Linie, "gegeben sind."

Wenn endlich die betrachtete Curve drei Doppelpuncte hat, so fallen die drei ersten Glieder der Gleichung (11.) weg und die Curve zerfält in drei gerade Linien, deren Gleichungen $y_{1}=0, y_{2}=0, y_{3}=0$ sind. Es seien diese drei geraden Linien dieselben, welche im vorhergehenden Falle mit diesen Symbolen bezeichnet wurden. Es entspricht jedem Puncte $\boldsymbol{P}$ ein Pol $\boldsymbol{p}$ unter Vermittelung der Gleichungen:

$$
\begin{array}{r}
0+\boldsymbol{Y}_{2} y_{3}+\boldsymbol{Y}_{3} y_{2}=0 \\
\boldsymbol{Y}_{1} y_{3}+0+\boldsymbol{Y}_{3} y_{1}=0, \\
\boldsymbol{Y}_{1} y_{2}+\boldsymbol{Y}_{2} y_{1}+0=0
\end{array}
$$

woraus sich zeigt, dafs jedem Puncte $\boldsymbol{P}$ auf einer Seite des durch die drei geraden Linien gebildeten Dreiecks der Pol $p$ entspricht, der harmonisch ist zu ihm und dem Punctenpaare, welches die in Rede stehende Seite des Dreiecks begrenzt. Durchschneidet man das Dreieck mit der geraden Linie $\boldsymbol{A}$ in drei Puncten $\boldsymbol{P}$, so wird der Kegelschnitt

18. $\alpha_{1} \alpha_{1} y_{1}^{2}+\alpha_{2} \alpha_{2} y_{2}^{2}+\alpha_{3} \alpha_{3} y_{3}^{2}-2 \alpha_{2} \alpha_{3} y_{2} y_{3}-2 \alpha_{3} \alpha_{1} y_{3} y_{1}-2 \alpha_{1} \alpha_{2} y_{1} y_{2}=0$ das Dreieck in den drei entsprechenden Polen berühren. Und diese Gleichung stellt, unter der Voraussetzung, dafs $\alpha_{1}, \alpha_{2}, \alpha_{3}$ beliebig variiren, alle Kegelschnitte dar, welche die Seiten des Dreiecks berühren. Zieht man die Gleichung (18.) von der (17.) ab, so erhält man $-\frac{2 \alpha_{2} \alpha_{3} y_{1}^{2}}{\pi}=0$; welches beweiset, dafs die Kegelschnitte (17. und 18.) in dem Berührungspuncte der geraden Linie $y_{1}=0$ eine 4punctige Berührung haben. Dieses läfst sich wie folgt zusammenfassen. Wenn man einen gegebenen Kegelschnitt und ein gegebenes Tangentenpaar desselben mit einer geraden Linie $L$ durchschneidet, so lassen sich zwei Kegelschnitte construiren, von welchen der eine den gegebenen Kegelschnitt in den beiden Schnittpuncten der geraden Linie $L$ und zugleich die Verbindungslinie $M$ der Tangirungspuncte des gegebenen Tangentenpares, der andere das Tangentenpaar in den Schnittpuncten der geraden Linie $L$ und sugleich die gerade Linie $M$ 
berührt. Diese beiden Kegelschnitte berühren sich vierpunctiy in demjenigen Puncte der geraden Linie M, welcher harmonisch ist su dem Schnittpuncte der Linien $L$ und $M$ und den Endpuncten der geraden Linie $M$.

6.

Den drei Systemen von Kegelschnitten (9.), welche die gegebene Curve dritter Ordnung $\varphi=0$ in drei verschiedenen Puncten berühren, ordnen sich auch alle diejenigen Kegelschnitte unter, welche die Curve einmal vierpunctig und das anderemal zweipunctig berühren. Diese Art von Kegelschnitten (9.) entsprechen denjenigen unter den durch (8.) dargestellten geraden Linien $\boldsymbol{A}$, welche die Curve berühren. Um die Berührungspuncte eines beliebigen dieser Kegelschnitte zu finden, nehme man ein Polenpaar $\boldsymbol{P}, \boldsymbol{p}$ an und schneide die Curve durch die gerade Linie $\boldsymbol{P}_{\boldsymbol{p}}$ in $\boldsymbol{q}$. Alsdann giebt es zwei Kegelschnitte, welche die Curve in $q$ zweipunctig berühren und von welchen der eine die Curve in $\boldsymbol{P}$, der andere in $\boldsymbol{p}$ vierpunclig berührt; und diese beiden Kegelschnitte gehören einem und demselben Systeme an. Es erhellet hieraus:

18. Da/s alle Kegelschnitte, welche eine Curve dritter Ordnung, einmal vierpunctiy, das anderemal zweipunctig berüleren, in drei Systeme zerfallen. Ein gegebener Kegelschnitt dieser Art gehört dem einen oder dem andern Systeme an, je nachdem der vierpunctige Berührungspunct und der Schnittpunct der Verbindungslinie der beiden Berührungspuncte und der Curve ein Polenpaar des einen oder des anderen Systems sind.

Ist der vierpunctige Berührungspunct gegeben und der zweipunctige zu suchen, so verbinde man den vierpunctigen Berührungspunct mit den drei ihm in den drei Systemen entsprechenden Polen durch drei gerade Linien. Diese drei geraden Linien schneiden die Curve in den gesuchten Puncten. Die Aufgabe läfst also drei Auflösungen zu. Ist dagegen der zweipunctige Berührungspunct gegeben und der vierpunctige Berührungspunct zu suchen, so ergeben sich 12 Auflösungen der Aufgabe. Man erhält dieselben, wenn man zu dem gegebenen zweipunctigen Berührungspunct die drei Pole in den drei Systemen construirt und von den letzteren die 12 Tangenten an die Curve zieht. Die Berührungspuncte sind dann die gesuchten Puncte.

Um analytisch die Kegelschnitte (9.) zu bestimmen, welche die gegebene Curve dritter Ordnung, einmal vierpunctig, das anderemal zweipunctig berühren, bleibt noch übrig, die Relation zwischen den drei Gröfsen $\alpha_{1}, \alpha_{2}, \alpha_{3}$ aufzustellen, welche Statt finden mufs, damit die gerade Linie, deren Gleichung 
$\alpha_{1} x_{1}+\alpha_{2} x_{2}+\alpha_{3} x_{3}=0$ ist, eine Tangente der Curve dritter Ordnung $p=0$ sei; oder mit andern Worten: Wenn die Gleichung einer beliebigen Curve dritter Ordnung in Punctcoordinaten gegeben ist, dieselbe durch Liniencoordinaten auszudrücken.

Diese Aufgabe läfst sich auf folgende Weise symmetrisch lösen. Man bilde die Determinante $A$ aus folgenden Gröfsen:

$$
\begin{array}{cccc}
\frac{\partial^{2} \varphi}{\partial x_{1}^{2}}, & \frac{\partial^{2} \varphi}{\partial x_{1} \partial x_{2}}, & \frac{\partial^{2} \varphi}{\partial x_{1} \partial x_{3}}, & \alpha_{1}, \\
\frac{\partial^{2} \varphi}{\partial x_{2} \partial x_{1}}, & \frac{\partial^{2} \varphi}{\partial x_{2}^{2}}, & \frac{\partial^{2} \varphi}{\partial x_{2} \partial x_{3}}, & \alpha_{2}, \\
\frac{\partial^{2} \varphi}{\partial x_{3} \partial x_{1}}, & \frac{\partial^{2} \varphi}{\partial x_{3} \partial x_{2}}, & \frac{\partial^{2} \varphi}{\partial x_{3}^{2}}, & \alpha_{3}, \\
\alpha_{1}, & \alpha_{2}, & \alpha_{3}, & 0 .
\end{array}
$$

Sie wird sowohl in Beziehung auf $x_{1}, x_{2}, x_{3}$, als in Beziehung auf die Gröfsen $\alpha_{1}, \alpha_{1}, \alpha_{3}$ homogen und vom zweiten Grade sein. Man betrachte ferner die 6 Producte $x_{1} x_{1}, x_{2} x_{2}, \ldots x_{1} x_{2}$ in den 6 Gleichungen

$$
\begin{gathered}
\frac{\partial \varphi}{\partial x_{1}}+\alpha_{1}=0, \quad \frac{\partial \varphi}{\partial x_{2}}+\alpha_{2}=0, \quad \frac{\partial \varphi}{\partial x_{3}}+\alpha_{3}=0, \\
x_{1}\left(\alpha_{1} x_{1}+\alpha_{2} x_{2}+\alpha_{3} x_{3}\right)=0, \\
x_{2}\left(\alpha_{1} x_{1}+\alpha_{2} x_{2}+\alpha_{3} x_{3}\right)=0, \\
x_{3}\left(\alpha_{1} x_{1}+\alpha_{2} x_{2}+\alpha_{3} x_{3}\right)=0,
\end{gathered}
$$

als die Unbekannten und löse diese lineären Gleichungen auf. Setzt man alsdann die Werthe der genannten 6 Producte in die Gleichung $\boldsymbol{A}=0$, in welcher dieselben lineär vorkommen, so hat man die gesuchte homogene Gleichung vom 6ten Grade in Beziehung auf die Liniencoordinaten $\alpha_{1}, \alpha_{2}, \alpha_{3}$. Dafs das System von 6 Gleichungen Statt findet, wenn $\alpha_{1} x_{1}+\alpha_{2} x_{2}+\alpha_{3} x_{3}=0$ die Gleichung der Tangente der Curve $\varphi=0$ in dem Puncte ist, dessen Coordinaten $x_{1}, x_{2}, x_{3}$ sind, ist einleuchtend. Es bleibt also noch nachzuweisen, dafs auch die Determinante $\Delta$ unter diesen Bedingungen verschwindet. $\mathrm{Zu}$ diesem Ende bemerke ich, dafs von den 4 Gleichungen

$$
\begin{aligned}
\frac{\partial^{2} \varphi}{\partial x_{1}^{2}} x_{1}+\frac{\partial^{2} \varphi}{\partial x_{1} \partial x_{2}} x_{2}+\frac{\partial^{2} \varphi}{\partial x_{1} \partial x_{3}} x_{3}+2 \alpha_{1} t & =\boldsymbol{A}_{1}, \\
\frac{\partial^{2} \varphi}{\partial x_{2} \partial x_{1}} x_{1}+\frac{\partial^{2} \varphi}{\partial x_{2}^{2}} x_{2}+\frac{\partial^{2} \varphi}{\partial x_{2} \partial x_{3}} x_{3}+2 \alpha_{2} t & =A_{2}, \\
\frac{\partial^{2} \varphi}{\partial x_{3} \partial x_{1}} x_{1}+\frac{\partial^{2} \varphi}{\partial x_{3} \partial x_{2}} x_{2}+\frac{\partial^{2} \varphi}{\partial x_{3}^{2}} x_{3}+2 \alpha_{3} t & =A_{3}, \\
\alpha_{1} x_{1}+\quad \alpha_{2} x_{2}+\quad \alpha_{3} x_{3} & =A_{4}
\end{aligned}
$$


die drei ersten in die drei ersten mit dem Factor 2 multiplicirten Gleichungen des obigen Systems und die letzte in die Gleichung der Tangente übergehen, wenn man $t=1$ und $A_{1}=A_{2}=A_{3}=A_{4}=0$ setzt. Betrachtet man aber die in diesen Gleichungen explicite und lineär vorkommenden Gröfsen $x_{1}, x_{2}, x_{3}, t$ als Unbekannten und löset sie nach diesen Unbekannten, z. B. nach $x_{1}$, auf, so erhält man eine Gleichung von der Form

$$
x_{1} A=a_{1} A_{1}+a_{2} A_{2}+a_{3} A_{3}+a_{4} A_{4} .
$$

Setzt man nun $A_{1}=A_{2}=A_{3}=A_{4}=0$, so verschwindet $A$; was zu beweisen war.

7.

Unter den drei Systemen von Kegelschnitten (9.), welche die gegebene Curve dritter Ordnung in drei verschiedenen Puncten berühren, finden sich auch solche Kegelschnitte, welche *mit der Curve eine 6punctige Berührung haben. Denn wenn man die gerade Linie $\boldsymbol{A}$ in die Lage einer Wendetangente bringt, welche, wie bekannt, der Curve in 3 Puncten $\boldsymbol{P}$ begegnet, die in einen zusammenfallen, so werden die diesen entsprechenden Puncte $p$, welche die Berührungspuncte der Kegelschnitte (9.) sind, ebenfalls in einen zusammenfallen; woraus eben eine 6punctige Berührung entsteht. Da aber jeder von den 9 Wendepuncten drei ihm entsprechende Pole hat, so hat eine Curve dritter Ordnung im Allgemeinen 27 Puncte $\pi$, in welchen sie von Kegelschnitten 6punctig berührt werden kann. Wenn man von einem der Wendepuncte $\delta$ die drei Tangenten an die Curve zieht, so werden die Tangirungspuncte $\alpha, \beta, \gamma$ die dem Wendepuncte $\delta$ entsprechenden Pole in den drei Systemen sein; was aus der in (\$. 2.) angegebenen Construction des einem gegebenen Puncte der Curve entsprechenden Poles erhellet. Diese drei Puncte $\alpha, \beta, \gamma$ liegen in einer und derselben geraden Linie. Denn bekanntlich liegen die 6 Berührungspuncte der Tangenten, welche von einem beliebigen Puncte an die Curve dritter Ordnung gezogen werden können, in einem Kegelschnitte. Rückt nun der beliebige Punct in einen Wendepunct, so fallen 3 von den Berührungspuncten in einen Punct der Wendetangente; weshalb die drei übrigen ebenfalls in einer geraden Linie liegen müssen.

19. Die Berührungspuncte der 27 Tangenten, welche von den Wendepuncten einer Curve dritter Ordnung an die Curve gezogen werden können, sind diejenigen Puncte $\pi$, in welchen Kegelschnitte die Curve Gpunctig berühren können. Die 28 Kegelschnitte, welche die Curce Gpunctig 
berühren, so wie die 27 Berührungspuncte $\pi$, zerfallen in drei Systeme von je 9 Kegelschnitten oder 9 Puncten. Ein beliebiger von diesen Kegelschnitten und sein Berülırungspunct gehören dem einen oder dem andern Systeme an, je nachdem der Berührungspunct und der Schnittpunct der Tangente in dem Berührungspuncte und der Curve, welcher ein Wendepunct ist, ein Polenpaar des einen oder des andern Systems bilden.

Um die Lage der 27 Puncte $\alpha, \beta, \gamma$ zu einander zu erforschen, mufs man die Lage der 9 Wendepuncte $\delta$ ins Auge fassen. Letztere läfst sich aus dem Satze von Poncelet: „dafs jede, durch zwei Wendepuncte der Curve dritter „Ordnung gelegte gerade Linie auch einen dritten Wendepunct der Curve trifft," auf folgende Art erkennen. Wenn $\delta_{1}, \delta_{4}, \delta_{7}$ drei, nicht in einer geraden Linie liegende Wendepuncte sind, so schneiden die geraden Linien $\delta_{4} \delta_{7}, \delta_{7} \delta_{1}, \delta_{1} \delta_{4}$ die Curve respective in drei neuen Wendepuncten $\delta_{2}, \delta_{5}, \delta_{8}$. Man überzeugt sich leicht, dafs diese letzteren ebenfalls micht in einer geraden Linie liegen. Denn lägen sie in einer geraden Linie, so hätte man folgende 4 Combinationen der 6 Wendepuncte, welche in einer geraden Linie liegen:

$$
\delta_{4} \delta_{7} \delta_{2}, \quad \delta_{7} \delta_{1} \delta_{5}, \quad \delta_{1} \delta_{4} \delta_{8}, \quad \delta_{2} \delta_{5} \delta_{s} .
$$

Verbindet man diese 6 Wendepuncte mit irgend einem der drei andern durch 6 gerade Linien, so müfste jede von diesen Linien die Curve in einem neuen Wendepuncte schneiden. Man erhielte also auf diese Weise statt der 9 Wendepuncte 13. Da nun die Wendepuncte $\delta_{2}, \delta_{5}, \delta_{8}$ nicht in einer geraden Linie liegen, so werden die geraden Linien $\delta_{5} \delta_{8}, \delta_{8} \delta_{2}, \delta_{2} \delta_{5}$ die Curve in den drei noch übrigen Wendepuncten $\delta_{3}, \delta_{6}, \delta_{9}$ schneiden. Erwägt man endlich, dafs die geraden Linien $\delta_{1} \delta_{2}, \delta_{4} \delta_{5}, \delta_{7} \delta_{8}$ die Curve nur in den Puncten $\delta_{3}, \delta_{6}, \delta_{9}$ schneiden können, so erhält man folgende Combinationen der Wendepuncte. welche auf einer geraden Linie liegen:

$$
\begin{array}{llll}
\delta_{1} \delta_{2} \delta_{3} \ldots & \delta_{4} \delta_{5} \delta_{6} \ldots & \delta_{7} \delta_{8} \delta_{9}, \\
\delta_{2} \delta_{4} \delta_{7} \ldots & \delta_{3} \delta_{5} \delta_{8} \ldots & \delta_{1} \delta_{6} \delta_{9}, \\
\delta_{5} \delta_{7} \delta_{1} \ldots & \delta_{6} \delta_{8} \delta_{2} \ldots & \delta_{4} \delta_{9} \delta_{3}, \\
\delta_{8} \delta_{1} \delta_{4} \ldots & \delta_{9} \delta_{2} \delta_{5} \ldots & \delta_{7} \delta_{3} \delta_{6} .
\end{array}
$$

Ich werde die den Wendepuncten $\delta_{1}, \delta_{2}, \ldots \delta_{9}$ entsprechenden Pole $\pi$

In dem ersten Systeme durch $\alpha_{1}, \alpha_{2}, \ldots \alpha_{9}$,

In dem zweiten Systeme durch $\beta_{1}, \beta_{2}, \ldots \beta_{9}$,

In dem dritten Systeme durch $\gamma_{1}, \gamma_{2}, \ldots \gamma_{9}$

bezeichnen. Wenn nun $\delta_{x} \delta_{\lambda} \delta_{\mu}$ irgend eine der angegebenen 12 Combinationen der Wendepuncte ist, welche in einer geraden Linie liegen, so sind 


$$
\alpha_{\alpha} \alpha_{\lambda} \delta_{\mu}, \quad \beta_{x} \beta_{\lambda} \delta_{\mu}, \quad \gamma_{x} \gamma_{\lambda} \delta_{\mu}
$$

drei Coordinaten von Puncten, welche ebenfalls in einer geraden Linie liegen. Denn wenn man ein Polenpaar $\alpha_{x} \delta_{x}$ mit einem zweiten Polenpaar $\alpha_{\lambda} \delta_{\lambda}$ desselben Systems durch zwei gerade Linien $\alpha_{x} \alpha_{\lambda}$ und $\delta_{x} \delta_{\lambda}$ verbindet, so schneiden sich die Verbindungslinien nach (N. 6.) in einem und demselben Puncte der Curve. Es schneidet aber die gerade Linie $\delta_{x} \delta_{\lambda}$ die Curve in $\delta_{\mu}$ : folglich liegen die Puncte $\alpha_{z}, \alpha_{\lambda}, \delta_{\mu}$ in einer und derselben Linie; was sich kurz so ausdrücken läfst:

20. Jede gerade Linie, welche swei Puncte $\pi$ aus demselben Systeme verbindet, schneidet die Curve dritter Ordnung in einem Wendepuncte; woraus folgt, da/s 108 mal swei Puncle $\pi$ und ein Wendepunct in einer geraden Linie liegen.

Wenn, wie oben, $\delta_{x} \delta_{\lambda} \delta_{\mu}$ irgend eine Combination der Wendepuncte ist, welche auf einer und derselben geraden Linie liegen, so ist $\alpha_{x} \beta_{\lambda} \gamma_{\mu}$ eine Combination von drei Puncten $\pi$, welche ebenfalls auf einer geraden Linie liegen. Denn wenn man ein Polenpaar $\alpha_{x} \delta_{x}$ mit einem Polenpaare $\beta_{\lambda} \delta_{\lambda}$ eines anderen Systems durch zwei gerade Linien $\delta_{x} \delta_{\lambda}$ und $\alpha_{x} \beta_{\lambda}$ verbindet, so schneiden dieselben nach (N. 14.) die Curve in einem Polenpaare $\delta_{\mu} \gamma_{\mu}$ des dritten Systems. Dieses, mit der obigen Bemerkung, dafs auch $\alpha_{x}, \beta_{x}, \gamma_{x}$ in einer geraden Linie liegen, verbunden, giebt folgenden Lehrsatz:

21. Jede gerade Linie, welche zwei Puncte $\pi$ verbindet, die verschiedenen Systemen angehören, schneidet die Curve dritter Ordnung in einem Puncte $\pi$ des dritten Systems. Es liegen also 81 mal drei Puncte $\pi$ auf einer geraden Linie *).

Da die 9 Puncte $\pi$ aus einem und demselben Systeme die den 9 Wendepuncten der Curve 3ter Ordnung in diesem Systeme entsprechenden Pole sind, so werden von den ersteren so oft 6 Puncte auf einem Kegelschnitt liegen, als von den letzteren 6 auf einem Linienpaare liegen. Hieraus folgt mit Rücksicht auf die angegebene Lage der Wendepuncte, der Lehrsatz:

22. Von den 9 Puncten $\pi$ eines und desselben Systems liegen 66 mal 6 Puncle auf einem Kegelschnitt.

*) Herr Professor Stciner giebt in dem Auszuge aus seiner am 27ten Nov. 1845 in der Akademie der Wissenschaften zu Berlin gehaltenen Vorlesung (in diesem Journal Bd. 32. S. 182) die Zahl der geraden Linien, von denen jede durch drei Puncte $\pi$ hindurchgeht, auf 108 an. Nach meiner Auseinandersetzung müssen einige von den 108 geraden $\mathrm{Li}-$ nien, welche dieser berühmte Geometer im Auge gehabt hat, zusammenfallen, so dafs nur 81 wirklich von einander verschiedene Linien übrig bleiben. 
Hieraus erhellet, dafs diese 9 Puncte nicht die Durchschnittspuncte zweier Curven dritter Ordnung sein können.

Wenn man in dem Lehrsatze (18.) für den ersten Kegelschnitt denjenigen auswählt, welcher in einem Puncte $\pi$ die Curve 6punctig berührt, so ergiebt sich folgende Eigenschaft dieser Puncte:

23. Alle Kegelschnitte, welche eine Curve dritter Ordnung in einem Puncte $\pi$ 3punctig berühren, schneiden die Curve in drei Puncten, in welchen ein anderer Kegelschnitt die Curve berührt; und umgekehrt:

24. Es giebt 9 Kegelschnitle, welche durch die drei Ecken eines der Curve dritter Ordnung einbeschriebenen Dreiecks hindurchgehen, dessen Seiten die Curve in drei in gerader Linie liegenden Puncten schneiden und in einem andern Puncte die Curve dreipunctig berühren. Der Ort dieser Berührungspuncte sind die 9 Puncte $\pi$, welche demselben Systeme angehören, dem das Dreieck angehört.•

Königsberg, im Juli 1847. 